\title{
Heavy quarkonium potential from Bethe-Salpeter wave function on the lattice
}

\author{
Taichi Kawanai ${ }^{1,2 *}$ and Shoichi Sasaki $i^{1,3 \dagger}$ \\ ${ }^{1}$ Theoretical Research Division, Nishina Center, RIKEN, Wako 351-0198, Japan \\ ${ }^{2}$ Department of Physics, Brookhaven National Laboratory Upton, NY 11973-5000, USA and \\ ${ }^{3}$ Department of Physics, Tohoku University, Sendai 980-8578, Japan
}

(Dated: October 30, 2018)

\begin{abstract}
We propose a novel method for the determination of the interquark potential together with quark "kinetic mass" $m_{Q}$ from the equal-time $Q \bar{Q}$ Bethe-Salpeter (BS) amplitude in lattice QCD. Our approach allows us to calculate spin-dependent $Q \bar{Q}$ potentials, e.g. the spin-spin potential, as well. In order to investigate several systematic uncertainties on such $Q \bar{Q}$ potentials, we carry out lattice QCD simulations using quenched gauge configurations generated with the single plaquette gauge action with three different lattice spacings, $a \approx 0.093,0.068$ and $0.047 \mathrm{fm}$, and two different physical volumes, $L \approx 2.2$ and $3.0 \mathrm{fm}$. For heavy quarks, we employ the relativistic heavy quark (RHQ) action which can control large discretization errors introduced by large quark mass $m_{Q}$. The spinindependent central $Q \bar{Q}$ potential for the charmonium system yields the "Coulomb plus linear" behavior with good scaling and small volume dependence. We explore the quark mass dependence over the wide mass range from the charm to beyond the bottom region, and then demonstrate that the spin-independent central $Q \bar{Q}$ potential in the $m_{Q} \rightarrow \infty$ limit is fairly consistent with the static $Q \bar{Q}$ potential obtained from Wilson loops. The spin-spin potential at finite quark mass provides a repulsive interaction with a finite range, which becomes narrower as the quark mass increases. We also discuss the applicability of the $1 / m_{Q}$ expansion approach for the spin-spin potential.
\end{abstract}

\section{INTRODUCTION}

The dynamics of heavy quarks having much larger masses than the QCD scale, $\Lambda_{\mathrm{QCD}}$, can be analyzed within the formalism of nonrelativistic quantum mechanics. In quark potential models, physical quantities such as mass spectra and decay rates of heavy quarkonium states are indeed calculated by solving the Schrödinger equation with heavy "constituent quark mass" [1]. The so-called Cornell potential is often adopted as an interquark potential between a heavy quark $(Q)$ and an anti-quark $(\bar{Q})[2]$.

The Cornell potential is consisted of a Coulomb part and a linear part as

$$
V(r)=-\frac{4}{3} \frac{\alpha_{s}}{r}+\sigma r
$$

where $\alpha_{s}$ is the strong coupling constant and $\sigma$ denotes the string tension [2]. The first term is generated by perturbative one-gluon exchange, while the linearly rising potential describes the phenomenology of confining quark interactions. Indeed confining nature of QCD is a key ingredient for understanding heavy quarkonium physics [2-6].

Spin-dependent potentials (spin-spin, tensor and spinorbit terms) appear as relativistic corrections to the spinindependent central potential in powers of the inverse of the heavy quark mass $m_{Q}[7,8]$. In potential models, their functional forms are basically determined by perturbative one-gluon exchange as the Fermi-Breit type

\footnotetext{
*Electronic address: kawanai@nt.phys.s.u-tokyo.ac.jp

${ }^{\dagger}$ Electronic address: ssasaki@nucl.phys.tohoku.ac.jp
}

potential $[7,8]$. For heavy quarkonia $\left(m_{Q}=m_{\bar{Q}}\right)$, the spin-dependent potentials are give by

$$
\begin{aligned}
& V_{\text {spin-dep }}=\frac{1}{m_{Q}^{2}}\left[\frac{32 \pi \alpha_{s}}{9} \delta(r) \mathbf{S}_{Q} \cdot \mathbf{S}_{\bar{Q}}\right. \\
+ & \left.\frac{4 \alpha_{s}}{r^{3}}\left(\frac{\left(\mathbf{S}_{Q} \cdot \mathbf{r}\right)\left(\mathbf{S}_{\bar{Q}} \cdot \mathbf{r}\right)}{r^{2}}-\frac{\mathbf{S}_{Q} \cdot \mathbf{S}_{\bar{Q}}}{3}\right)+\left(\frac{2 \alpha_{s}}{r^{3}}-\frac{\sigma}{2 r}\right) \mathbf{L} \cdot \mathbf{S}\right],
\end{aligned}
$$

where $\mathbf{S}=\mathbf{S}_{Q}+\mathbf{S}_{\bar{Q}}$. Although there are many successes in the conventional charmonium spectrum, many of the newly discovered charmonium-like mesons, named as " $X Y Z$ " mesons, could not be simply explained by quark potential models [9]. However, the phenomenological spin-dependent potentials determined by perturbative method would have validity only at short distances and also in the vicinity of the heavy quark mass limit. We thus consider that properties of higher-mass charmonium states predicated in quark potential models may suffer from large uncertainties.

In order to make more accurate theoretical predictions in quark potential models, the reliable interquark potential directly derived from first principles QCD is highly desired. One of the major successes of lattice QCD is to qualitatively justify the Coulomb plus linear potential by the static heavy quark potential obtained from Wilson loops [10]. Indeed, the $Q \bar{Q}$ potential between infinitely heavy quark and antiquark has been precisely determined by lattice QCD in the past few decades [10-15].

The relativistic corrections to the static potential are classified in powers of $1 / m_{Q}$ within a framework called potential nonrelativistic QCD (pNRQCD) [16]. The lattice determination of the spin-dependent terms has been carried out within the quenched approximation in the 1980s [17-22] and the 1990s [23, 24], and it has been extended to dynamical QCD simulations [25, 26]. However, 
these earlier studies did not enable us to determine the functional forms of the spin-dependent terms due to large statistical errors.

Recently, corrections of the leading and next-to-leading order in the $1 / m_{Q}$ expansion to the static $Q \bar{Q}$ potential have been successfully calculated in quenched lattice QCD with high accuracy by using the multilevel algorithm $[27,28]$. However calculation of the realistic charmonium potential in lattice QCD within Wilson loop formalism is still rather difficult. The inverse of the charm quark mass would be obviously far outside the validity region of the $1 / m_{Q}$ expansion. Furthermore, a spin-spin potential determined at $\mathcal{O}\left(1 / m_{Q}^{2}\right)[27,28]$, which provides an attractive interaction for the higher spin states, yields wrong mass ordering among hyperfine multiplets. The higher order corrections beyond the next-to-leading order are required to correctly describe the conventional heavy quarkonium spectrum even for the bottom quarks with the $Q \bar{Q}$ potentials obtained from this approach. In addition, practically, the multilevel algorithm employed in Refs. [27, 28] is not easy to be implemented in dynamical lattice QCD simulations.

Under these circumstances, we propose a novel method to determine the interquark potential using lattice QCD in this paper. The interquark potential is defined by the equal-time and Coulomb gauge Bethe-Salpeter (BS) amplitude through an effective Schrödinger equation. This is a variant of the mehod originally applied for the hadron-hadron interaction [29, 30], and enables us to determine both spin-independent and spin-dependent interquark potentials, at heavy, but finite quark mass. These potentials implicitly account for all orders of $1 / m_{Q}$ corrections [31]. Furthermore, there is no restriction to dynamical calculation within this method.

This paper is organized as follows. In Sec. II, we briefly review the methodology utilized in this paper to calculate the spin-independent and spin-dependent $Q \bar{Q}$ potentials with the finite quark mass in lattice $\mathrm{QCD}$ simulations. Sec. III contains details of our Monte Carlo simulations and some basic results. In Sec. IV, we show numerical results of the quark kinetic mass $m_{Q}$, the spin-independent central and spin-spin potentials. The spin-independent part of the resulting $Q \bar{Q}$ potential exhibits a good scaling behavior and small volume dependence. We also discuss several systematic uncertainties on the interquark potentials obtained from the BS amplitude. In Sec. V, in order to demonstrate the feasibility of the new approach, we show that the interquark potential calculated by the BS amplitude smoothly approaches the static $Q \bar{Q}$ potential from Wilson loops in the infinitely heavy quark limit. We also discuss an issue on the spin-spin potential in the conventional $1 / m_{Q}$ expansion approach. In Sec. VI, we summarize and discuss all results and future perspectives.

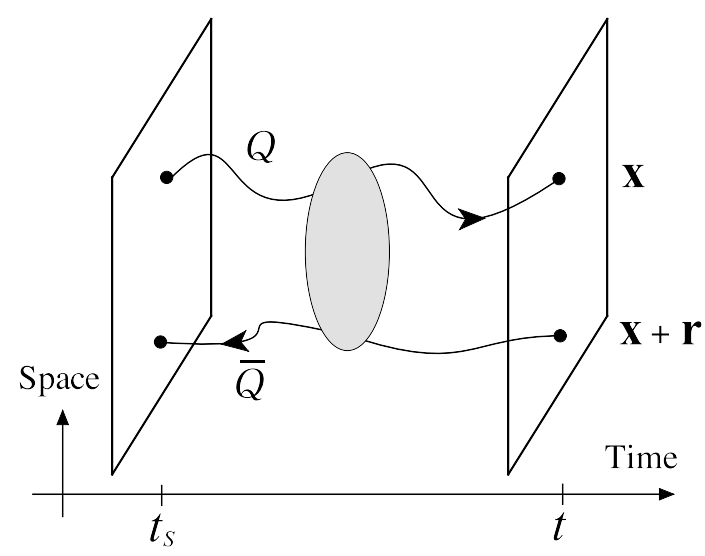

FIG. 1: A schematic illustration of the four-point correlation function for the $Q \bar{Q}$ system. Solid lines indicate quark propagators of a heavy quark and a heavy antiquark, located at $\mathbf{x}$ and $\mathbf{x}+\mathbf{r}$ at sink, respectively. At the source $\left(t_{S}\right)$ and $\operatorname{sink}(t)$, the gauge field configurations are fixed to the Coulomb gauge.

\section{FORMALISM}

In this section, we will briefly review the new method utilized here to calculate the interquark potential at finite quark mass. Our proposed method for a new determination of the interquark potential in lattice QCD is based on the same idea originally applied for the nuclear force [29,30], where the hadron-hadron potential is defined through the equal-time BS amplitude [29, 30, 3239]. Here we call this method as BS amplitude method. The quark kinetic mass $m_{Q}$, which is a key ingredient in the BS amplitude method applied to the $Q \bar{Q}$ system, is simultaneously determined through the large-distance behavior in the spin-dependent part of the interquark potential with the help of the measured hyperfine splitting energy of $1 S$ states in heavy quarkonia [31, 40].

\section{A. Equal-time $Q \bar{Q}$ BS wave function}

A gauge-invariant definition of the equal-time $Q \bar{Q} \mathrm{BS}$ amplitude for quarkonium states is given by

$$
\phi_{\Gamma}(\mathbf{r})=\sum_{\mathbf{x}}\left\langle 0|\bar{Q}(\mathbf{x}) \Gamma \mathcal{M}(\mathbf{x}, \mathbf{x}+\mathbf{r}) Q(\mathbf{x}+\mathbf{r})| Q \bar{Q} ; J^{P C}\right\rangle,
$$

where $\mathbf{r}$ is the relative coordinate of two quarks at a certain time slice, and $\Gamma$ is any of the 16 Dirac $\gamma$ matrices $[41,42]$. A summation over spatial coordinates $\mathbf{x}$ projects on a state with zero total momentum. $\mathcal{M}$ is a path-ordered product of gauge links. The $\mathbf{r}$-dependent amplitude, $\phi_{\Gamma}(\mathbf{r})$, is here called BS wave function. In the Coulomb or Landau gauge, the BS wave function can be simply evaluated with $\mathcal{M}=1$. Hereafter, we consider the Coulomb gauge BS wave function [42].

The Coulomb gauge BS wave function can be extracted from the following four-point correlation function that is 
also schematically depicted in Fig. 1:

$$
\begin{aligned}
& G_{\Gamma}\left(\mathbf{r}, t, t_{\mathrm{s}}\right) \\
= & \sum_{\mathbf{x}, \mathbf{x}^{\prime}, \mathbf{y}^{\prime}}\left\langle 0\left|\bar{Q}(\mathbf{x}, t) \Gamma Q(\mathbf{x}+\mathbf{r}, t)\left(\bar{Q}\left(\mathbf{x}^{\prime}, t_{\mathrm{s}}\right) \Gamma Q\left(\mathbf{y}^{\prime}, t_{\mathrm{s}}\right)\right)^{\dagger}\right| 0\right\rangle \\
= & \sum_{\mathbf{x}} \sum_{n} A_{n}\langle 0|\bar{Q}(\mathbf{x}) \Gamma Q(\mathbf{x}+\mathbf{r})| n\rangle e^{-M_{n}^{\Gamma}\left(t-t_{\mathrm{s}}\right)}
\end{aligned}
$$

where the gauge field configurations are fixed to the Coulomb gauge at both source $\left(t_{\mathrm{s}}\right)$ and sink $(t)$ locations. At source location, both quark and antiquark fields are separately averaged in space as wall sources. The constant amplitude $A_{n}$ is a matrix element defined as $A_{n}=\sum_{\mathbf{x}^{\prime}, \mathbf{y}^{\prime}}\left\langle n\left|\left(\bar{Q}\left(\mathbf{x}^{\prime}\right) \Gamma Q\left(\mathbf{y}^{\prime}\right)\right)^{\dagger}\right| 0\right\rangle . \quad M_{n}^{\Gamma}$ denotes a rest mass of the $n$-th quarkonium state $|n\rangle$ in a given $J^{P C}$ channel. Suppose that $\left|t-t_{\mathrm{s}}\right| / a \gg 1$ is satisfied, the four-point correlation function is dominated by the ground state as

$$
G_{\Gamma}\left(\mathbf{r}, t, t_{\mathrm{s}}\right) \stackrel{t \gg t_{\mathrm{s}}}{\longrightarrow} A_{0} \phi_{\Gamma, 0}(\mathbf{r}) e^{-M_{0}^{\Gamma}\left(t-t_{\mathrm{s}}\right)},
$$

where $M_{0}^{\Gamma}$ is the rest mass of the ground state and the $\mathbf{r}$-dependent amplitude $\phi_{\Gamma, 0}(\mathbf{r})$ corresponds to the Coulomb gauge BS wave function for the ground state. For instance, when $\Gamma$ is chosen to be $\gamma_{5}$ for the pseudoscalar (PS) channel $\left(J^{P C}=0^{-+}\right)$and $\gamma_{i}$ for the vector $(\mathrm{V})$ channel $\left(J^{P C}=1^{--}\right)$in the charm sector, $M_{0}^{\mathrm{PS}}$ and $M_{0}^{\mathrm{V}}$ correspond to the rest masses of the $\eta_{c}$ and $J / \psi$ ground states, respectively. They can be read off from the asymptotic large-time behavior of the two-point correlation functions. Hereafter, we omit the index 0 from $\phi_{\Gamma, 0}(\mathbf{r})$ and simply call it the BS wave function.

\section{B. Interquark potential defined from BS wave function}

The BS wave function satisfies an effective Schrödinger equation with a nonlocal and energy-independent interquark potential $U[29,43,44]$ :

$$
-\frac{\nabla^{2}}{2 \mu} \phi_{\Gamma}(\mathbf{r})+\int d r^{\prime} U\left(\mathbf{r}, \mathbf{r}^{\prime}\right) \phi_{\Gamma}\left(\mathbf{r}^{\prime}\right)=E_{\Gamma} \phi_{\Gamma}(\mathbf{r}),
$$

where the reduced mass $\mu$ of the quarkonium $(Q \bar{Q})$ system is given by a half of the quark kinetic mass $m_{Q}$. The energy eigenvalue $E_{\Gamma}$ of the stationary Schrödinger equation is supposed to be $M_{\Gamma}-2 m_{Q}$. If the relative quark velocity $v=\left|\nabla / m_{Q}\right|$ is small as $v \ll 1$, the nonlocal potential $U$ can generally expand in terms of the velocity $v$ as

$$
\begin{aligned}
& U\left(\mathbf{r}^{\prime}, \mathbf{r}\right)=\left\{V(r)+V_{\mathrm{S}}(r) \mathbf{S}_{Q} \cdot \mathbf{S}_{\bar{Q}}\right. \\
& \left.\quad+V_{\mathrm{T}}(r) S_{12}+V_{\mathrm{LS}}(r) \mathbf{L} \cdot \mathbf{S}+\mathcal{O}\left(v^{2}\right)\right\} \delta\left(\mathbf{r}^{\prime}-\mathbf{r}\right),
\end{aligned}
$$

where $S_{12}=\left(\mathbf{S}_{Q} \cdot \hat{r}\right)\left(\mathbf{S}_{\bar{Q}} \cdot \hat{r}\right)-\mathbf{S}_{Q} \cdot \mathbf{S}_{\bar{Q}} / 3$ with $\hat{r}=\mathbf{r} / r, \mathbf{S}=$ $\mathbf{S}_{Q}+\mathbf{S}_{\bar{Q}}$ and $\mathbf{L}=\mathbf{r} \times(-i \nabla)[29]$. Here, $V, V_{\mathrm{S}}, V_{\mathrm{T}}$ and $V_{\mathrm{LS}}$ represent the spin-independent central, spin-spin, tensor and spin-orbit potentials, respectively. Remark that the energy dependence on the interquark potential appear at $\mathcal{O}\left(v^{2}\right)$. For an estimation of the $\mathcal{O}\left(v^{2}\right)$ corrections, it is necessary to calculate the BS wave function of higherlying charmonium states, e.g. the $2 S$ charmonium state. Such study is beyond scope of the present paper [45].

The relativistic corrections to the kinetic term have been estimated using the relativistic kinematics in Ref. [44]. Although the short-range behavior of interquark potential is slightly influenced by this modification, it is indeed small for the heavy quarks such as the charm quark. Therefore, we here consider the nonrelativistic Schrödinger equation with spin-dependent corrections up to $\mathcal{O}\left(v^{2}\right)$.

In this paper, we focus only on the $S$-wave charmonium states $\left(\eta_{c}\right.$ and $\left.J / \psi\right)$. We perform an appropriate projection with respect to the discrete rotation as

$$
\phi_{\Gamma}^{A_{1}^{+}}(\mathbf{r})=\frac{1}{24} \sum_{\mathcal{R} \in O_{h}} \phi_{\Gamma}\left(\mathcal{R}^{-1} \mathbf{r}\right),
$$

where $\mathcal{R}$ denotes 24 elements of the cubic point group $O_{h}$. This projection provides the BS wave function projected in the $A_{1}^{+}$representation. This projected BS wave function corresponds to the $S$-wave in continuum theory at low energy [46]. We simply denote the $A_{1}^{+}$projected BS wave function by $\phi_{\Gamma}(r)$ hereafter.

The stationary Schrödinger equation for the projected BS wave function $\phi_{\Gamma}(r)$ is reduced to

$$
\left\{-\frac{\nabla^{2}}{m_{Q}}+V(r)+\mathbf{S}_{Q} \cdot \mathbf{S}_{\bar{Q}} V_{\mathrm{S}}(r)\right\} \phi_{\Gamma}(r)=E_{\Gamma} \phi_{\Gamma}(r) .
$$

The spin operator $\mathbf{S}_{Q} \cdot \mathbf{S}_{\bar{Q}}$ can be easily replaced by expectation values $-3 / 4$ and $1 / 4$ for the PS and V channels, respectively. We here essentially follow usual nonrelativistic potential models, where the $J / \psi$ state is assumed to be purely composed of the $1 S$ wave function. Within our proposed method, this assumption can be verified by evaluating the size of a mixing between $1 S$ and $1 D$ wave functions in principle.

Both spin-independent and dependent part of the central interquark potentials can be separately evaluated through a linear combination of Eq. (9) for PS and V channels as

$$
\begin{aligned}
V(r) & =E_{\text {ave }}+\frac{1}{m_{Q}}\left\{\frac{3}{4} \frac{\nabla^{2} \phi_{\mathrm{V}}(r)}{\phi_{\mathrm{V}}(r)}+\frac{1}{4} \frac{\nabla^{2} \phi_{\mathrm{PS}}(r)}{\phi_{\mathrm{PS}}(r)}\right\} \\
V_{\mathrm{S}}(r) & =E_{\mathrm{hyp}}+\frac{1}{m_{Q}}\left\{\frac{\nabla^{2} \phi_{\mathrm{V}}(r)}{\phi_{\mathrm{V}}(r)}-\frac{\nabla^{2} \phi_{\mathrm{PS}}(r)}{\phi_{\mathrm{PS}}(r)}\right\}
\end{aligned}
$$

where $E_{\text {ave }}=M_{\text {ave }}-2 m_{Q}$ and $E_{\text {hyp }}=M_{\mathrm{V}}-M_{\mathrm{PS}}$. The mass $M_{\text {ave }}$ denotes the spin-averaged mass as $\frac{1}{4} M_{\mathrm{PS}}+$ $\frac{3}{4} M_{\mathrm{V}}$. The derivative $\nabla^{2}$ is defined by the discrete Laplacian. For other spin-dependent potentials (the tensor potential $V_{\mathrm{T}}$ and the spin-orbit potential $V_{\mathrm{LS}}$ ), this approach, in principle, enables us to access them by considering $P$-wave quarkonium states such as the $\chi_{c}\left(0^{++}\right.$, 
$\left.1^{++}\right)$and $h_{c}\left(1^{+-}\right)$states, which must leave contributions of $V_{\mathrm{T}}$ and $V_{\mathrm{LS}}$ to Eq. (9).

\section{Quark kinetic mass in BS amplitude method}

The definition of the interquark potentials in Eq. (10) and (11) involves unknown information of the quark mass $m_{Q}$ that appears in the kinetic energy term of the effective Schrödinger equation (Eq. (6) or (9)). This is an essential issue on the BS amplitude method when we apply it to the $Q \bar{Q}$ system. Needless to say, the original work, where the BS amplitude method was advocated and applied for the nuclear force [29], does not share the same issue since the single-nucleon mass can be measured by the standard hadron spectroscopy.

In the initial attempt [44], the quark kinetic mass $m_{Q}$ was approximately evaluated by one-half of the vector quarkonium mass $M_{\mathrm{V}} / 2$. However, such an approximate treatment is too crude to define a proper interquark potential, which could be smoothly connected to the static $Q \bar{Q}$ potential from Wilson loops in the $m_{Q} \rightarrow \infty$ limit. Indeed, it is worth noting that the Coulombic binding energy is of order of $m_{Q}$. We may alternatively determine the quark mass from the gauge dependent pole mass, which can be measured by the quark two-point function in the Landau gauge. In this case, we are faced with a difference between the Coulomb and Landau gauges. In Ref. [31], we have solved this issue by proposing a novel idea to determine the quark kinetic mass $m_{Q}$ selfconsistently within the $B S$ amplitude approach. Let us shortly review the new idea as follows in this subsection.

We start from the spin-spin potential given by Eq. (11). The hyperfine splitting energy, $E_{\mathrm{hyp}}=M_{\mathrm{V}}-M_{\mathrm{PS}}$, appeared in Eq. (11) can be measured by the standard hadron spectroscopy. Under a simple but reasonable assumption:

$$
\lim _{r \rightarrow \infty} V_{\mathrm{S}}(r)=0
$$

which implies that there is no long-range correlation and no irrelevant constant term in the spin-dependent potential. Eq. (11) is thus rewritten as

$$
m_{Q}=\lim _{r \rightarrow \infty} \frac{-1}{E_{\mathrm{hyp}}}\left\{\frac{\nabla^{2} \phi_{\mathrm{V}}(r)}{\phi_{\mathrm{V}}(r)}-\frac{\nabla^{2} \phi_{\mathrm{PS}}(r)}{\phi_{\mathrm{PS}}(r)}\right\} .
$$

This suggests that the quark kinetic mass can be read off from the long-distance asymptotic values of the difference of quantum kinetic energies (the 2nd derivative of the BS wave function) in $\mathrm{V}$ and PS channels. This idea has been numerically tested, and the assumption of Eq. (12) is indeed appropriate in QCD [31].

As a result, we can self-consistently determine both the spin-independent potential $V(r)$ and spin-spin potential $V_{\mathrm{S}}(r)$, and also the quark kinetic mass $m_{Q}$ within a single set of four-point correlation functions $G_{\Gamma}\left(\mathbf{r}, t, t_{\mathrm{S}}\right)$ with $\Gamma=\mathrm{PS}$ and $\mathrm{V}$.
TABLE I: Simulation paramters of quenched ensembles. Lattice spacing $a$ indicates the approximate value with the Sommer scale $\left(r_{0}=0.5 \mathrm{fm}\right)$ input. The table also lists the number of gauge configurations to be analyzed.

\begin{tabular}{ccccccc}
\hline \hline Label & $L^{3} \times T$ & $\beta$ & $a[\mathrm{fm}]$ & $a^{-1}[\mathrm{GeV}]$ & $L a[\mathrm{fm}]$ & Statistics \\
\hline FI & $48^{3} \times 96$ & 6.47 & 0.0469 & 4.2 & 2.3 & 100 \\
ME & $32^{3} \times 64$ & 6.2 & 0.0677 & 2.9 & 2.2 & 150 \\
CO & $24^{3} \times 48$ & 6.0 & 0.0931 & 2.1 & 2.2 & 300 \\
LA & $32^{3} \times 48$ & 6.0 & 0.0931 & 2.1 & 3.0 & 150 \\
\hline \hline
\end{tabular}

TABLE II: The hopping parameter $\kappa_{Q}$ and RHQ parameters $\left(\nu, r_{s}, c_{B}\right.$ and $\left.c_{E}\right)$ for the charm quark on all four ensembles.

\begin{tabular}{ccccccc}
\hline \hline Label & $\beta$ & $\kappa_{Q}$ & $\nu$ & $r_{s}$ & $c_{B}$ & $c_{E}$ \\
\hline FI & 6.47 & 0.11729 & 1.029 & 1.131 & 1.700 & 1.562 \\
ME & 6.2 & 0.11035 & 1.050 & 1.185 & 1.898 & 1.710 \\
CO, LA & 6.0 & 0.10072 & 1.088 & 1.273 & 2.194 & 1.932 \\
\hline \hline
\end{tabular}

\section{LATTICE SETUP AND HEAVY QUARKONIUM MASS}

\section{A. Quenched gauge ensembles}

In order to understand the systematics of the BS amplitude method, we first calculate the interquark potential for the charmonium system in quenched lattice QCD simulations using several ensembles (three different lattice spacings, $a \approx 0.093,0.068$ and $0.047 \mathrm{fm}$, and two different physical volumes, $L a \approx 2.2$ and $3.0 \mathrm{fm}$ ). The gauge configurations are generated with the single plaquette gauge action. All lattice spacings are set by the Sommer scale $r_{0}=0.5 \mathrm{fm}$ [47].

Three smaller volume ensembles with fixed physical volume $(L a \approx 2.2 \mathrm{fm})$ are mainly employed to test a scaling behavior toward the continuum limit: these are the finer lattice ensembles (FI) on a $24^{3} \times 48$ lattice at $\beta=6.47$, the medium ones (ME) on a $32^{3} \times 64$ lattice at $\beta=6.2$ and the coarser ones (CO) on a $24^{3} \times 48$ lattice at $\beta=6.0$. A supplementary data calculated on the larger volume ensembles (LA) on a $32^{3} \times 48$ lattice at $\beta=6.0$ are used for a check of possible finite volume effects. The number of configurations analyzed is $\mathcal{O}(100-300)$. The gauge configurations are fixed to the Coulomb gauge for calculations of the BS amplitude. Simulation parameters and the number of sampled gauge configurations are summarized in Table I.

\section{B. Parameters of RHQ action}

The heavy quark propagators are computed using the RHQ action that has five parameters $\kappa_{Q}, \nu, r_{s}, c_{B}$ and $c_{E}$ [48]. The RHQ action used here is a variant of the Fermilab approach, and can control large discretization errors introduced by large quark mass [49] 

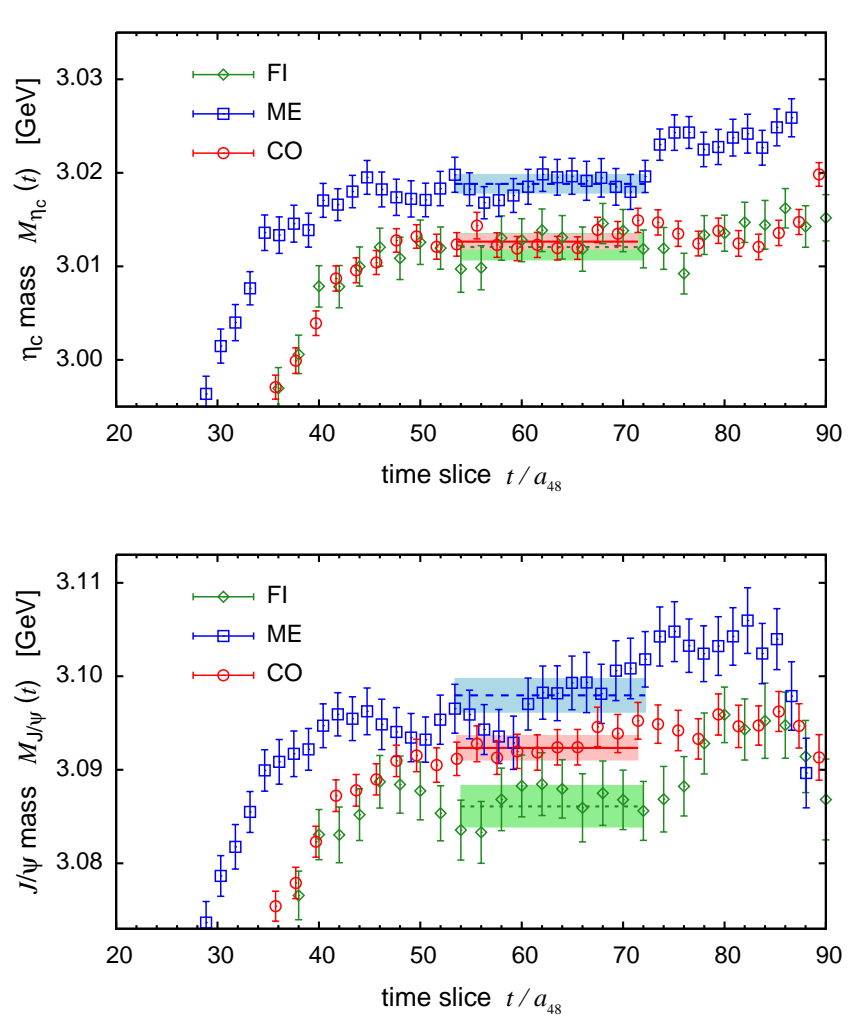

FIG. 2: Effective mass plots for the $\eta_{c}$ (upper) and $J / \psi$ (lower). Three different symbols indicate results obtained on the CO (circles), ME (squares) and FI (diamonds) ensembles. The horizontal axis is plotted in units of $a_{48}$, which is the lattice spacing of the FI ensembles. Solid, dashed and dotted lines represent the fit results for effective mass calculated on the CO, ME and FI ensembles, respectively. Shaded bands denote fit ranges and statistical errors estimated by the jackknife method.

(See also Refs. [50-52]). The hopping parameter is chosen to reproduce the experimental spin-averaged mass of $1 S$ charmonium states $M_{\text {ave }}^{\exp }=3.0678(3) \mathrm{GeV}$ [53] at each lattice spacing. The five RHQ parameters are basically determined by one-loop perturbative calculations [54]. The parameter sets of the RHQ action in quenched simulations at three lattice spacings are summarized in Table II.

We calculate quark propagators with a wall source. Dirichlet boundary conditions are imposed for the time direction. In order to investigate the energy-momentum dispersion relation, we also employ a gauge invariant Gaussian-smeared source [55] for the standard two-point correlation function with four finite momenta: $a \mathbf{p}=$ $2 \pi / L \times(1,0,0),(1,1,0),(1,1,1)$ and $(2,0,0)$.

\section{Effective mass from two point function}

The mass $M_{\Gamma}$ of the charmonium states $(\Gamma=$ PS and $\mathrm{V}$ ) is extracted by the two-point function. When a sepa- ration between a quark and an anti-quark at the sink is set to be zero $(\mathbf{r}=0)$, the four-point correlation functions $G_{\Gamma}\left(\mathbf{r}, t, t_{\mathrm{s}}\right)$ defined in Eq. (4) simply reduces the usual two-point function $G_{\Gamma}\left(t, t_{\mathrm{s}}\right)$ with a wall source. The effective mass functions are then defined as

$$
M_{\Gamma}(t)=\log \frac{G_{\Gamma}\left(t, t_{\mathrm{s}}\right)}{G_{\Gamma}\left(t+1, t_{\mathrm{s}}\right)} .
$$

Fig. 2 shows the effective mass plots of the $1 S$ charmonium states $\left(\eta_{c}\right.$ and $\left.J / \psi\right)$, calculated on three ensembles (FI, ME, CO). Each effective mass plot shows a reasonable plateau. We estimate the $\eta_{c}$ and $J / \psi$ masses by a constant fit to the plateaus over time ranges shown in Table 2. A correlation between masses measured at various time slices is took into account by using a covariance matrix in the constant fit. A inversion of covariance matrix is performed once for average and used for each jackknife block. The statistical uncertainties indicated by shaded bands in Fig. 2 is estimated by the jackknife method. For all ensembles, we basically take similar fitting ranges in the same units, indicated by shaded bands in Fig. 2. All fit results are summarized in Table III. Also, values of spin-averaged mass $M_{\text {ave }}$ and hyperfine splitting energy $E_{\text {hyp }}$ are quoted in Table III. Note that we simply neglect the disconnected diagrams in calculations of both four-point and two-point correlation functions for the $\eta_{c}$ and $J / \psi$ states in our simulations.

We observe that on the FI ensembles the data of fourpoint and two-point correlation functions at different time slices are highly correlated. This strong correlation between the time slices becomes more pronounced when we calculate the interquark potential from the BS wave function. In the analysis of the interquark potential, we have to somehow reduce the correlation, which makes the covariance matrix singular, in order to get a reasonable value of $\chi^{2} /$ d.o.f. during the fitting. Therefore we will use the data points at even number time slices for evaluation of the BS wave function on the FI ensembles. Note that the effective mass for the FI ensembles was evaluated only with even number time slices to perform a consistent analysis (see Fig. 2, where the FI data points are appeared only at even number time slices).

The spin-averaged masses measured on the ME and CO (LA) ensembles slightly deviate from experimental data (see Table III). This implies that our calibration for the hopping parameters of the charm sector is not precise enough. Then, strictly speaking, a systematic uncertainty due to tuning the charm quark mass is larger than the statistical one. However its accuracy is still enough to study the interquark potential for the charmonium in this quenched studies. As we will discuss later, although the discrepancy among the spin-averaged masses given at three lattice spacings is kept less than one percent, the resulting quark kinetic masses are fairly consistent with each other albeit with rather large statistical errors.

For the hyperfine splitting energy, results obtained from our quenched simulations reproduce only $65-70 \%$ of experimental value $M_{\text {hyp }}^{\text {exp }}=113.2(7) \mathrm{MeV}$ (See also 
TABLE III: Fitted masses of $1 S$ charmonium states, their spin-averaged masses and hyperfine splitting energies obtained with the same fit range on all four ensembles. Results are tabulated in units of GeV. The hopping parameter $\kappa$ on each ensembles is chosen approximately to reproduce the experimental spin-averaged mass of $1 \mathrm{~S}$ charmonium states $M_{\mathrm{ave}}^{\exp }=3.0678(3) \mathrm{GeV}[53]$.

\begin{tabular}{|c|c|c|c|c|c|c|c|}
\hline \multirow[t]{2}{*}{ Label } & \multirow{2}{*}{$\begin{array}{c}\text { fit range } \\
{\left[t_{\min } / a: t_{\max } / a\right]}\end{array}$} & \multicolumn{2}{|c|}{$\eta_{c}$ mass } & \multicolumn{2}{|c|}{$J / \psi$ mass } & \multirow{2}{*}{$\begin{array}{l}\text { spin-averaged mass } \\
M_{\text {ave }}[\mathrm{GeV}]\end{array}$} & \multirow{2}{*}{$\begin{array}{l}\text { hyperfine splitting energy } \\
E_{\text {hyp }}[\mathrm{GeV}]\end{array}$} \\
\hline & & $M_{\eta_{c}}[\mathrm{GeV}]$ & $\chi^{2} /$ d.o.f. & $M_{J / \psi}[\mathrm{GeV}]$ & $\chi^{2} /$ d.o.f. & & \\
\hline FI & {$[54: 72]$} & $3.0121(14)$ & 0.66 & $3.0861(22)$ & 0.62 & $3.0676(20)$ & $0.0741(11)$ \\
\hline ME & {$[37: 50]$} & $3.0188(10)$ & 0.55 & $3.0980(18)$ & 0.93 & $3.0783(15)$ & $0.0773(11)$ \\
\hline $\mathrm{CO}$ & {$[27: 36]$} & $3.0126(8)$ & 1.65 & $3.0923(13)$ & 1.02 & $3.0724(11)$ & $0.0795(8)$ \\
\hline LA & {$[30: 39]$} & $3.0120(8)$ & 0.98 & $3.0907(13)$ & 0.75 & $3.0710(10)$ & $0.0790(8)$ \\
\hline
\end{tabular}

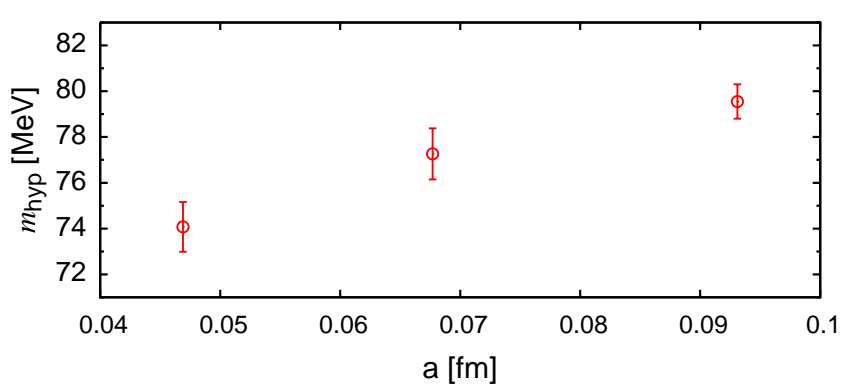

FIG. 3: The lattice spacing dependence of hyperfine splitting energies calculated on the FI, ME and CO ensembles. Results are shown in units of $\mathrm{MeV}$ as a function of lattice spacing in units of fm.

Ref. [40]). As shown in Fig. 3, the data points exhibit a slight linear dependence of the lattice spacing. We consider that the lattice spacing dependence of the hyperfine splitting energy is mainly caused by a remnant $\mathcal{O}(a)$ discretization error, rather than the issues related to calibration of the precise measurement at the charm quark mass, since our RHQ action with one-loop perturbative coefficients do not fully improve the leading discretization error. From this observation, we speculate that the discretization effect would be non-negligible for the spinspin potential, which is highly sensitive to the hyperfine splitting energy by the definition given in Eq. (11).

In the continuum theory, a relativistic particle, of which the rest mass is $m$, moving with spatial momentum $\mathbf{p}$ obeys the energy-momentum dispersion relation as $E^{2}=m^{2}+\mathbf{p}^{2}$. However, in lattice QCD, the dispersion relation deviates from the continuum one due to the presence of lattice discretization corrections as

$$
(a E)^{2}=(a m)^{2}+c_{\text {eff }}^{2}(a \mathbf{p})^{2}+c^{\prime}|a \mathbf{p}|^{4}+\mathcal{O}\left(a^{6}\right),
$$

where the spatial momentum is given by $a \mathbf{p}=\frac{2 \pi \mathbf{n}}{L}, \mathbf{n} \in$ $\mathbb{Z}^{3}$ in a finite $L^{3}$ box with periodic boundary conditions. A coefficient $c_{\text {eff }}^{2}$ appearing in the second term is squared effective speed of light. In the continuum limit $a \rightarrow 0$, $c_{\text {eff }}$ should be unity and higher order corrections vanish. If the discretization effect due to finite lattice spacing is well under control by using an improved action, $c_{\text {eff }}^{2}$ is supposed to remain approximately unity.
Fig. 4 shows the energy-momentum dispersion relations for the $\eta_{c}$ and $J / \psi$ states, and their spin-averaged one on the $\mathrm{CO}$ ensembles as a typical example. Our data up to spatial momenta of $\mathbf{n}^{2}=4$ well reproduces the continuum dispersion relation and resulting $c_{\text {eff }}^{2}$ is consistent with unity within error bars. The other ensembles also provide the similar results.

\section{DETERMINATION OF INTERQUARK POTENTIAL}

\section{A. $Q \bar{Q}$ BS wave function}

In Fig. 5, we show the reduced $Q \bar{Q}$ BS wave functions $u_{\Gamma}(r)=r \phi_{\Gamma}(\mathbf{r})$ of $1 S$ charmonium states $\left(\eta_{c}\right.$ and $J / \psi$ states), calculated on the FI ensembles. The normalized $Q \bar{Q}$ BS wave function with the definition given in Eq. (3) can be evaluated by the following ratio of four-point correlation functions $G_{\Gamma}\left(\mathbf{r}, t, t_{\mathrm{s}}\right)$ at large Euclidean time:

$$
\tilde{\phi}_{\Gamma}(\mathbf{r})=\frac{\phi_{\Gamma}(\mathbf{r})}{\sum_{\mathbf{r}}\left\{\phi_{\Gamma}(\mathbf{r})\right\}^{2}}=\lim _{\left|t-t_{\mathrm{s}}\right| \rightarrow \infty} \frac{G_{\Gamma}\left(\mathbf{r}, t, t_{\mathrm{s}}\right)}{\sum_{\mathbf{r}}\left\{G_{\Gamma}\left(\mathbf{r}, t, t_{\mathrm{s}}\right)\right\}^{2}} .
$$

We take an average of this ratio with respect to the time slice by a weighted sum in the range, where the effective mass of the $1 S$ charmonium states exhibits a clear plateau behavior. Here, the normalized wave function $\tilde{\phi}_{\Gamma}(r)$ satisfies a condition $\sum \tilde{\phi}_{\Gamma}^{2}=1$. We use the reduced wave function $u_{\Gamma}(r)$ for displaying the spatial distribution of the BS wave function. We focus on data points taken at $\mathbf{r}$ vectors, which are multiples of three directions, $(1,0,0)$ (on-axis), $(1,1,0)$ (off-axis I) and $(1,1,1)$ (off-axis II).

As shown in Fig. 5, the $Q \bar{Q}$ BS wave function projected in the $A_{1}^{+}$representation, which corresponds to the $S$-wave in the continuum theory, is certainly isotropic as was expected. In general, the breaking of rotational symmetry is one of major artifacts associated with the discretization error. However, there is no sufficient difference between the $Q \bar{Q}$ BS wave functions calculated along three different directions. It suggests that the discretization effect due to finite lattice spacing would be considerably small. Indeed, the $Q \bar{Q} \mathrm{BS}$ wave function of 

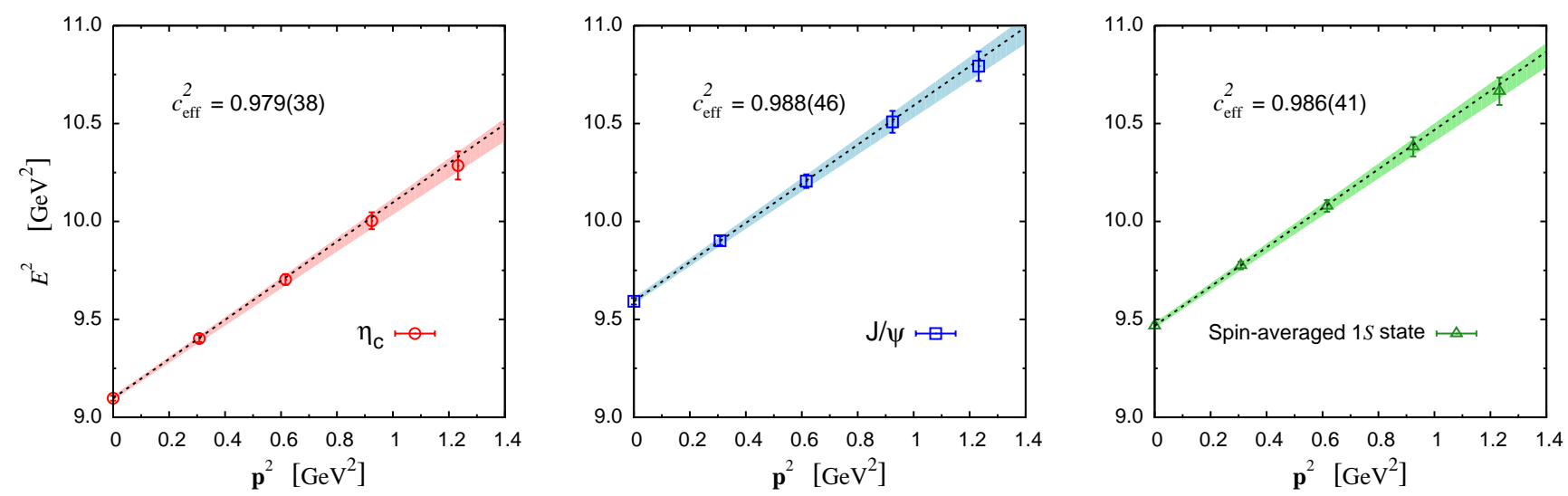

FIG. 4: Check of the energy-momentum dispersion relation $E^{2}\left(\mathbf{p}^{2}\right)=M^{2}+c_{\mathrm{eff}}^{2} \mathbf{p}^{2}$ for the $\eta_{c}$ (left), $J / \psi$ (center) and spinaveraged $1 S$ state (right) calculated on the $\mathrm{CO}$ ensembles, as typical examples. By the linear fit to data points calculated with various spatial momenta including zero momentum, the effective speed of light is obtained. Values of the squared effective speed of light $c_{\text {eff }}^{2}$ are quoted in each panel. Shaded bands indicate statistical uncertainties in fitting, estimated by the jackknife method. For comparison, the continuum dispersion relation $\left(c_{\mathrm{eff}}^{2}=1\right)$ is denoted as the dashed lines in each panel.

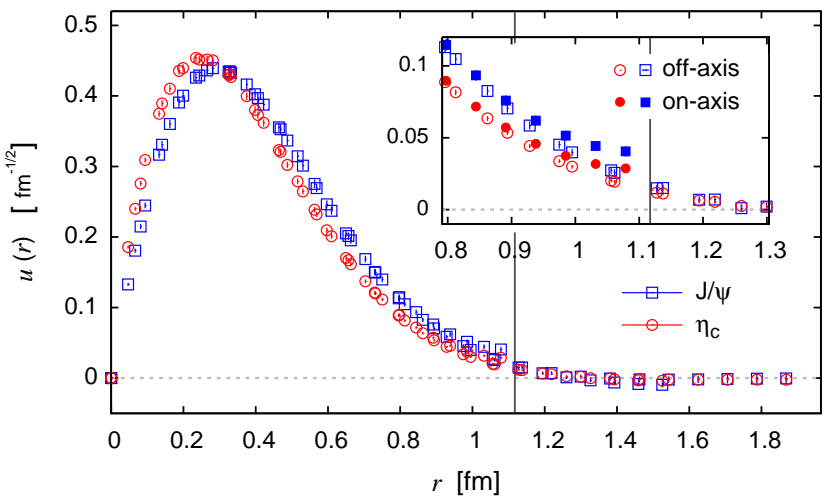

FIG. 5: The $Q \bar{Q}$ BS wave functions of the $\eta_{c}$ (circles) and $J / \psi$ (squares) states calculated using the FI ensembles $(a \approx$ $0.047 \mathrm{fm}$ ), shown as a function of the spatial distance $r$. The data points are taken at $\mathbf{r}$ vectors, which are multiples of three directions, $(1,0,0),(1,1,0)$ and $(1,1,1)$. A vertical solid line marks the position of a half of the lattice size $(L a / 2 \approx 1.1 \mathrm{fm})$. The inset shows a magnified view of the wave functions around $r \approx L a / 2$ and filled symbols in the inset represent the data points taken along the on-axis direction.

the $\eta_{c}$ state shows a good scaling behavior as shown in Fig. 6. All data of the $\eta_{c}$ wave function obtained from three ensembles (LI, ME, CO) clearly fall onto a single curve. Nothing changes for the $J / \psi$ wave function.

Fig. 5 and 6 show that the $Q \bar{Q}$ BS wave functions of $1 S$ charmonium states vanish for $r \lesssim 1 \mathrm{fm}$ and eventually fit into the lattice volume utilized here. Such localized wave functions indicate that the $1 S$ charmonium states are bound states. Therefore, the finite volume effect on the interquark potential is expected to be small, and the spatial extent of the present lattice size $(L a \approx 2.2 \mathrm{fm})$ is likely to be large enough to study the $1 S$ charmonium

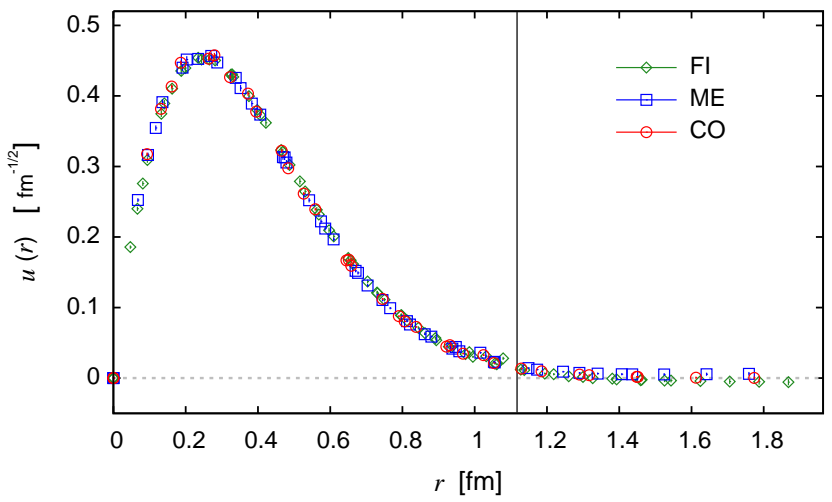

FIG. 6: The $Q \bar{Q}$ BS wave functions of the $\eta_{c}$ state calculated on the FI, ME and CO ensembles. A vertical solid line marks the position of a half of the lattice size $(L a / 2 \approx 1.1 \mathrm{fm})$.

states. However, there is still some caveat for the on-axis data. The vanishing point $r \sim 1 \mathrm{fm}$ is very close to a half of the spatial extent of the present lattice size, which is depicted as a solid vertical line in Fig. 5 and 6. A wrapround effect would be set in the on-axis direction near the spatial boundary. In fact, the on-axis data marginally deviates from the off-axis data at around $r \sim 1 \mathrm{fm}$ (See the inset of Fig. 5).

\section{B. Discrete Laplacian operator}

We next discuss choices of the discrete Laplacian operator $\nabla_{\text {lat }}^{2}$, which is built in the definition of the interquark potential. The discrete Laplacian operator on lattice can be naively defined with nearest neighbor points in the Cartesian coordinate system as below, called $x$-Laplacian 

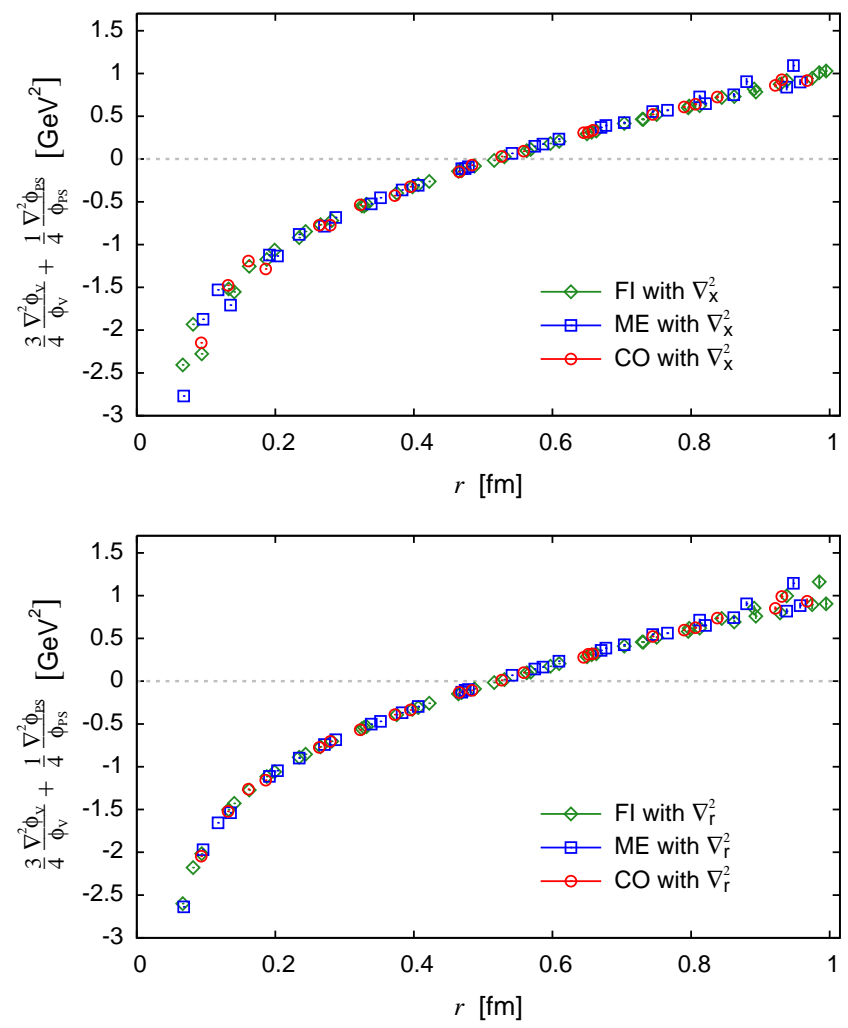

FIG. 7: The spin-averaged ratios $\frac{3}{4} \nabla_{\text {lat }}^{2} \phi_{\mathrm{V}} / \phi_{\mathrm{V}}+$ $\frac{1}{4} \nabla_{\text {lat }}^{2} \phi_{\mathrm{PS}} / \phi_{\mathrm{PS}}$ as functions of the spatial distance $r$, which are calculated with the discrete $x$-Laplacian $\nabla_{\mathbf{x}}^{2}$ (upper) and discrete $r$-Laplacian $\nabla_{\mathbf{r}}^{2}$ (lower) operators. Three different symbols denote results obtained from three different ensembles: the CO (circles), ME (squares) and FI (diamonds) ensembles.

in this paper:

$$
\begin{aligned}
\nabla_{\mathbf{x}}^{2} \phi_{\Gamma}(\mathbf{r}) & =\sum_{\hat{\mathbf{i}}=\hat{x}, \hat{y}, \hat{z}} \frac{1}{a^{2}}\left\{\phi_{\Gamma}(\mathbf{r}+\hat{\mathbf{i}}) a+\phi_{\Gamma}(\mathbf{r}+\hat{\mathbf{i}} a)-2 \phi_{\Gamma}(\mathbf{r})\right\} \\
& =\nabla_{\mathrm{cont}}^{2} \phi_{\Gamma}(\mathbf{r})+\mathcal{O}\left(a^{2}\right)
\end{aligned}
$$

where $\nabla_{\text {cont }}^{2}$ is the continuum Laplacian operator. A discretization error introduced by the discrete derivative operator starts at $\mathcal{O}\left(a^{2}\right)$.

In order to clarify the systematic uncertainties of the discrete Laplacian, we focus on a spin-averaged ratio

$$
\mathcal{V}(r)=\frac{3}{4} \nabla_{\text {lat }}^{2} \phi_{\mathrm{V}} / \phi_{\mathrm{V}}+\frac{1}{4} \nabla_{\text {lat }}^{2} \phi_{\mathrm{PS}} / \phi_{\mathrm{PS}},
$$

which is associated with the spin-independent interquark potential apart from the vertical scale and offset. This spin-averaged ratio is suitable for understanding the systematic uncertainty on the discrete Laplacian. Statistical and systematic uncertainties of $\mathcal{V}(r)$ are relatively small due to absence of the quark kinetic mass, whose determination introduces large statistical fluctuation, in comparison to the potential itself. In other wards, this spin-averaged ratio is independent of the definition of the quark mass.

The upper panel of Fig. 7 shows the spin-averaged ratios $\mathcal{V}(r)$ calculated with the $x$-Laplacian using the FI, $\mathrm{ME}$ and $\mathrm{CO}$ ensembles. Although the ratios in the upper panel of Fig. 7 show more or less the same scaling behavior as found in the $Q \bar{Q}$ BS wave function, some multiple-valuedness, which represents the rotational symmetry breaking, appears at short distances and also at long distances. Near the spatial boundary $r \gtrsim 0.9 \mathrm{fm}$, this unexpected sign of the rotational symmetry breaking could be explained by the finite volume effect. In practice, we naturally have a difficulty to obtain reliable data at long distances because the $Q \bar{Q}$ wave functions of $1 S$ charmonium state are quickly dumped (Fig. 5 and 6 ) and signal-to-noise ratio turns out to be worse in the ratio $\nabla^{2} \phi_{\Gamma} / \phi_{\Gamma}$. However, we have already seen in Fig. 5 that the on-axis data slightly deviates from the off-axis data near the spatial boundary in the BS wave function. Such small finite size effect should be inherited in the interquark potential.

On the other hand, the multivalued spin-averaged ratio appeared in $r \lesssim 0.3 \mathrm{fm}$ is inconsistent with no sign of the rotational symmetry breaking in the BS wave function. This implies that the multiple-valuedness appeared near the origin in the spin-averaged ratios is mainly stemming from the discretization artifact of the Laplacian operator.

To reduce the possible discretization error at short distances, we try to consider the following discrete Laplacian operator defined in the discrete polar coordinate, called r-Laplacian:

$$
\begin{aligned}
\nabla_{\mathbf{r}}^{2} \phi_{\Gamma}(r)= & \frac{2}{r} \frac{\phi_{\Gamma}(r+\tilde{a})-\phi_{\Gamma}(r-\tilde{a})}{2 \tilde{a}} \\
& +\frac{\phi_{\Gamma}(r+\tilde{a})+\phi_{\Gamma}(r-\tilde{a})-2 \phi_{\Gamma}}{\tilde{a}^{2}} \\
= & \frac{2}{r} \frac{\partial}{\partial r} \phi_{\Gamma}(r)+\frac{\partial^{2}}{\partial r^{2}} \phi_{\Gamma}(r)+\mathcal{O}\left(\tilde{a}^{2}\right),
\end{aligned}
$$

where $r$ is the absolute value of the relative distance as $r=|\mathbf{r}|=\sqrt{x^{2}+y^{2}+z^{2}}$ and $\tilde{a}$ is a distance between grid points along differentiate directions. We compute the ratio $\mathcal{V}(r)$ with the polar Laplacian in three directions: the on-axis, off-axis I and off-axis II, where the effective grid spacings correspond to $\tilde{a}=a, \sqrt{2} a, \sqrt{3} a$, respectively. Note here that the discretization errors induced by $\nabla_{\mathbf{r}}^{2}$ in the off-axis I and II directions are two and three time as much as in the on-axis direction, respectively.

In Eq. (19), we assume that the $Q \bar{Q}$ BS wave function $\phi_{\Gamma}(\mathbf{r})$ depends only on the distance $r$, namely $\phi_{\Gamma}(\mathbf{r})$ is isotropic. This is a reasonable assumption for the data shown in Fig. 6. Small, but visible effects of rotational symmetry breaking on $\phi_{\Gamma}(\mathbf{r})$ is simply encoded into discretization effects on the ratio $\mathcal{V}(r)$. They must vanishes at the continuum limits $a \rightarrow 0$ and infinite volume limits $L \rightarrow \infty$.

The derivative term of $\nabla_{\text {lat }}^{2} \phi_{\Gamma}(r)$ evaluated with both 
discrete Laplacian operators, $\nabla_{\mathbf{x}}^{2}$ and $\nabla_{\mathbf{r}}^{2}$, must essentially give the same answer in $a \rightarrow 0$ and $L \rightarrow \infty$.

The spin-averaged ratio $\mathcal{V}(r)$ calculated with $\nabla_{\mathbf{r}}$ is shown in the lower panel of Fig. 7. A shape of the ratio obtained with the polar Laplacian is highly improved to satisfy the single-valuedness at short distances. Similar to the $Q \bar{Q}$ BS wave function, the data points of the spinaveraged ratio calculated on three different ensembles fall onto a single curve at short distances. The rotational symmetry is also effectively recovered.

These results suggest us that the discrete polar Laplacian operator is better than the naive one to evaluate the interquark potential from the $S$-wave $Q \bar{Q}$ BS wave function. On the other hand, the rotational symmetry breaking observed at long distances due to the finite volume is not cured, or rather slightly enhanced. In this work, the $r$-Laplacian operator $\nabla_{\mathbf{r}}^{2}$ is used for the 2nd derivatives $\nabla^{2}$. Then, the subscript $\mathbf{r}$ on $\nabla_{\mathbf{r}}^{2}$ is omitted hereafter.

\section{Time average}

The ratios of $\nabla_{\text {lat }}^{2} \phi_{\Gamma} / \phi_{\Gamma}$ at each spatial point $r$, shown in Fig. 7, are actually evaluated by a weighted sum of the corresponding ratios of $\nabla_{\text {lat }}^{2} G_{\Gamma}(\mathbf{r}, t) / G_{\Gamma}(\mathbf{r}, t)$ with respect to the time slice in the range, where the effective mass plot of the two-point function shows the plateau. To resolve the strong correlations between data at different time slices, we take into account the full covariance matrix during the averaging process over the time slice.

Fig. 8 shows time dependence of the the spin-averaged ratio $\mathcal{V}(r)=\frac{3}{4} \nabla^{2} \phi_{\mathrm{V}} / \phi_{\mathrm{V}}+\frac{1}{4} \nabla^{2} \phi_{\mathrm{PS}} / \phi_{\mathrm{PS}}$ and the difference of ratios $\mathcal{V}_{S}(r)=\nabla^{2} \phi_{\mathrm{V}} / \phi_{\mathrm{V}}-\nabla^{2} \phi_{\mathrm{PS}} / \phi_{\mathrm{PS}}$ calculated on the FI ensembles at charm quark mass as a typical example. Both quantities are needed to calculate the spinindependent central, spin-spin potentials and quark kinetic mass through Eq. (10), (11) and (13), respectively. In Fig. 8, they exhibit reasonably long plateaus, and the asymptotic values at given $r$ can be read off from them. Solid lines represent average values over the plateau region. Shaded bands denote statistical errors estimated by the jackknife method. There is no qualitative difference in the results obtained from the other ensembles (ME, $\mathrm{CO}$ and LA).

\section{Quark kinetic mass}

In this subsection, we present the determination of the quark kinetic mass within the BS amplitude method. A precise determination of the quark kinetic mass is required for high-accuracy measurement of the interquark potentials. In Fig. 9, we plot the difference divided by the hyperfine splitting energy at charm quark mass as a function of the spatial distance $r$. At a glance, the value of $-\left(\nabla^{2} \phi_{\mathrm{V}} / \phi_{\mathrm{V}}-\nabla^{2} \phi_{\mathrm{PS}} / \phi_{\mathrm{PS}}\right) / E_{\mathrm{hyp}}$, which appears in the r.h.s. of Eq. (13), certainly reaches a nonzero constant value at large distances and it turns out to be the value of the quark mass $m_{Q}$.

Practically the quark mass $m_{Q}$ is obtained by a constant fit to an asymptotic value over the range, where $V_{\mathrm{S}}(r)$ should vanish, taking into account the full covariance matrix during the fitting process. In this study, such constant fit is individually performed to the three data sets obtained from three directions: on-axis, off-axis I and off-axis II. A difference of the quark masses obtained from the different directions exposes the size of the possible finite size effect. We will quote it as a systematic error on the quark kinetic mass. We finally take an average of the resulting masses over the three directions. The results of the quark kinetic mass are summarize in Table IV and also in Fig. 10.

As we mentioned in subsection IV B, the discretization error introduced by the discrete Laplacian operator defined in Eq. (19) along the off-axis I and II directions are expected to be greater than that of the on-axis data. Indeed we cannot obtain a reasonable value of $\chi^{2} /$ d.o.f. from the constant fits onto the off-axis data for the $\mathrm{CO}$ and LA ensembles, which are generated at the coarsest lattice spacing (See Table IV). We also find that the lattice spacing dependence of the quark kinetic mass determined from the on-axis data is observed to be the smallest in Fig. 10. For the CO and LA ensembles, we therefore prefer to use the on-axis data solely in the analysis of the quark kinetic mass, instead of the averaged value over three directions, in the following discussion.

Final results on the quark kinetic mass calculated at the three different lattice spacings (FI, $\mathrm{ME}$ and $\mathrm{CO}$ ensembles) show a good agreement with each other. The largest difference among three results is only less than a few \%. Although our calibration of the RHQ parameters is not precise enough as described in subsection III C, a good scaling is again observed in the quark kinetic mass within the current statistical precision.

According to a direct comparison between results obtained in two different lattice volumes ( $L a \approx 3.0 \mathrm{fm}$ and $2.2 \mathrm{fm})$ at the coarsest lattice spacing ( $\mathrm{CO}$ and LA ensembles), the systematic uncertainty due to the finite volume effect is estimated as a few \% level. We confirm that there is no significant volume effect in our evaluation of the quark kinetic mass even for the on-axis data.

\section{E. Spin-independent interquark potential}

Using the quark mass determined in previous subsection, we can calculate both the spin-independent central and spin-spin potential obtained from a set of the $Q \bar{Q} \mathrm{BS}$ wave function $\phi_{\Gamma}(r)$ with $\Gamma=$ PS and V, through Eq. (10) and (11). The BS wave functions $\phi_{\Gamma}(r)$ are defined only by the ground state contributions of the $r$-dependent amplitude $G_{\Gamma}(r, t)$. We determine the values of interquark potentials $V(r)$ and $V_{\mathrm{S}}(r)$ by averaging over appropriate time-slice range (See subsection IV C). 

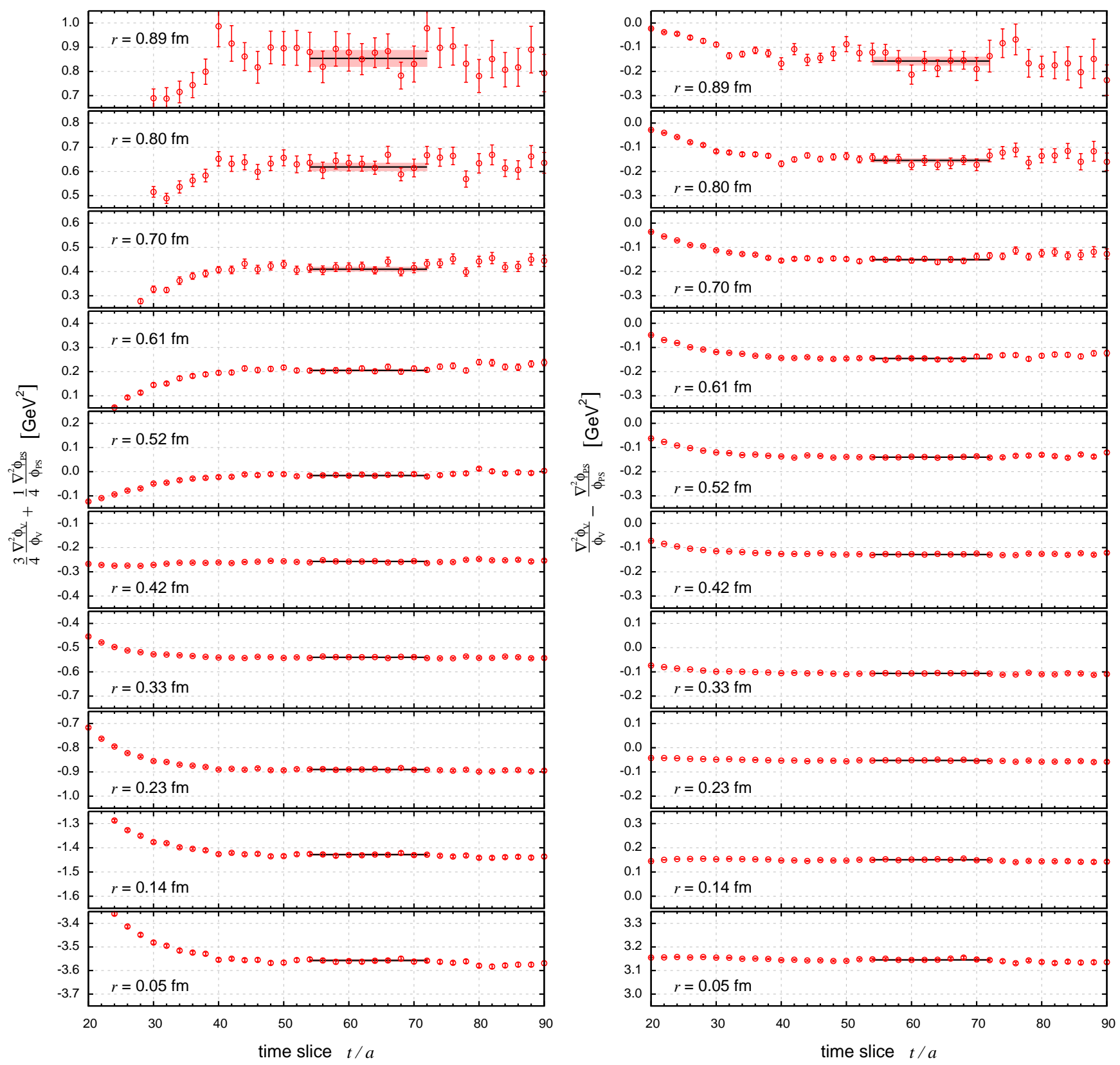

FIG. 8: The time slice dependence of the spin-averaged ratio $\mathcal{V}(r)$ (left) and the difference of ratios $\mathcal{V}_{S}(r)$ (right) at various distances over the range $0.05 \mathrm{fm} \leq r \leq 0.89 \mathrm{fm}$, which are calculated on the FI ensembles at the charm quark mass as a typical example.

TABLE IV: Summary of the quark kinetic masses determined along three different directions (on-axis, off-axis I and II) with the fit range $\left[r_{\min } / \tilde{a}: r_{\text {mas }} / \tilde{a}\right]$ for all four ensembles.

\begin{tabular}{|c|c|c|c|c|c|c|c|c|c|c|}
\hline \multirow[b]{2}{*}{ Label } & \multicolumn{3}{|c|}{ direction $(1,0,0), \tilde{a}=a$} & \multicolumn{3}{|c|}{ direction $(1,1,0), \tilde{a}=\sqrt{2} a$} & \multicolumn{3}{|c|}{ direction $(1,1,1), \tilde{a}=\sqrt{3} a$} & \multirow{2}{*}{$\begin{array}{c}\text { average } \\
m_{Q}[\mathrm{GeV}]\end{array}$} \\
\hline & fit range & $m_{Q}[\mathrm{GeV}]$ & $\chi^{2} /$ d.o.f. & fit range & $m_{Q}[\mathrm{GeV}]$ & $\chi^{2} /$ d.o.f. & fit range & $m_{Q}[\mathrm{GeV}]$ & $\chi^{2} /$ d.o.f. & \\
\hline FI & {$[14: 20]$} & $1.982(56)$ & 1.11 & [10:14] & $1.997(52)$ & 1.68 & {$[8: 11]$} & $2.030(50)$ & 1.62 & $2.013(43)$ \\
\hline $\mathrm{ME}$ & {$[9: 14]$} & $1.967(50)$ & 0.60 & {$[7: 10]$} & $1.990(60)$ & 0.44 & {$[6: 8]$} & 1.984( & 0.34 & $1.980(55)$ \\
\hline $\mathrm{CO}$ & {$[7: 10]$} & 1. & 0 & {$[5:$} & 1.8 & 4.55 & {$[4$} & $1 . \varepsilon_{0}$ & 7.13 & 1.90 \\
\hline LA & {$[7: 13]$} & $1.874(39)$ & 0.86 & {$[5: 9]$} & $1.917(37)$ & 1.29 & {$[4: 7]$} & $1.892(33)$ & 4.12 & $1.895(32)$ \\
\hline
\end{tabular}




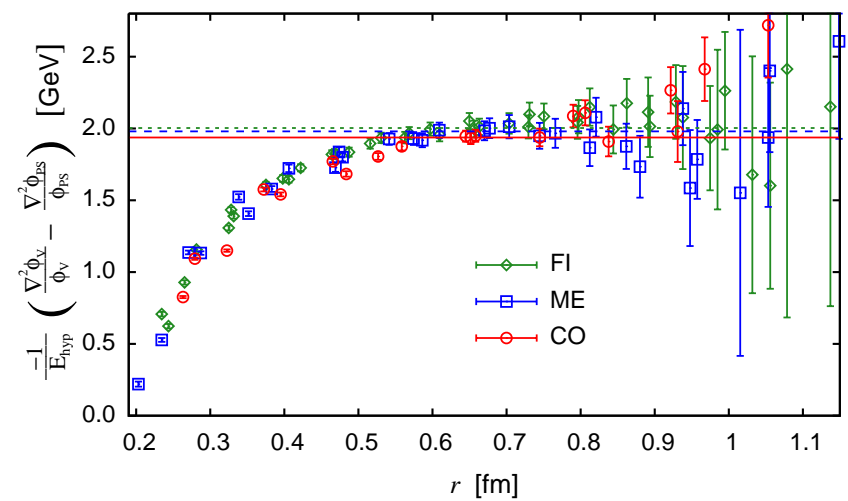

FIG. 9: The determination of the quark kinetic mass within the BS amplitude method. The values of $-\left(\nabla^{2} \phi_{\mathrm{V}} / \phi_{\mathrm{V}}-\right.$ $\left.\nabla^{2} \phi_{\mathrm{PS}} / \phi_{\mathrm{PS}}\right) / E_{\mathrm{hyp}}$ as a function of the spatial distance $r$ are shown in this figure. Circle, square and diamond symbols denote results calculated on the FI, ME and $\mathrm{CO}$ ensembles, respectively. The quark kinetic masses $m_{Q}$ are evaluated from the long-distance asymptotic values of $-\left(\nabla^{2} \phi_{\mathrm{V}} / \phi_{\mathrm{V}}-\right.$ $\left.\nabla^{2} \phi_{\mathrm{PS}} / \phi_{\mathrm{PS}}\right) / E_{\mathrm{hyp}}$. Horizontal solid (CO), dashed (ME) and dotted (FI) lines indicate results of the quark kinetic masses, which are determined by a weighted average of data points in the range $0.6 \mathrm{fm} \lesssim r \lesssim 1.0 \mathrm{fm}$ as described in text.

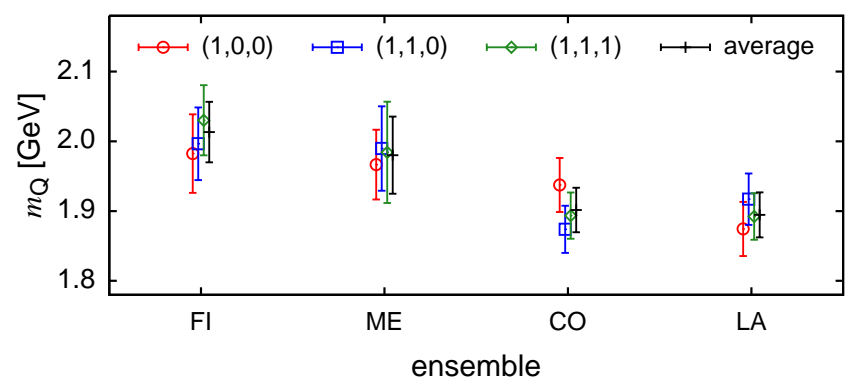

FIG. 10: The quark kinetic mass calculated on all four ensembles. Circle, square and diamond symbols denote results calculated in the on-axis, off-axis I and off-axis II directions, respectively. Their averaged values are indicated by cross symbols.

The upper panel of Fig. 11 shows all results of the spin-independent potential $V(r)$ at charm quark mass, that are calculated on three ensembles (FI, ME and CO) with fixed physical volume. For clarity of the figure, the constant energy shift $E_{\text {ave }}$, which corresponds to a value of $M_{\text {ave }}-2 m_{Q}<0$, is not subtracted in Fig. 11. As expected, the resulting spin-independent central potential $V(r)$ with finite quark mass exhibits the linearly rising potential at large distances and the Coulomb-like potential at short distances.

In the upper panel of Fig. 11, the data points of the interquark potentials measured at different lattice spacings collapse on a single curve. This would indicate that simulations at the gauge couplings $\beta=6 / g^{2}=6.0,6.2$ and 6.47 are already in the asymptotic scaling region.
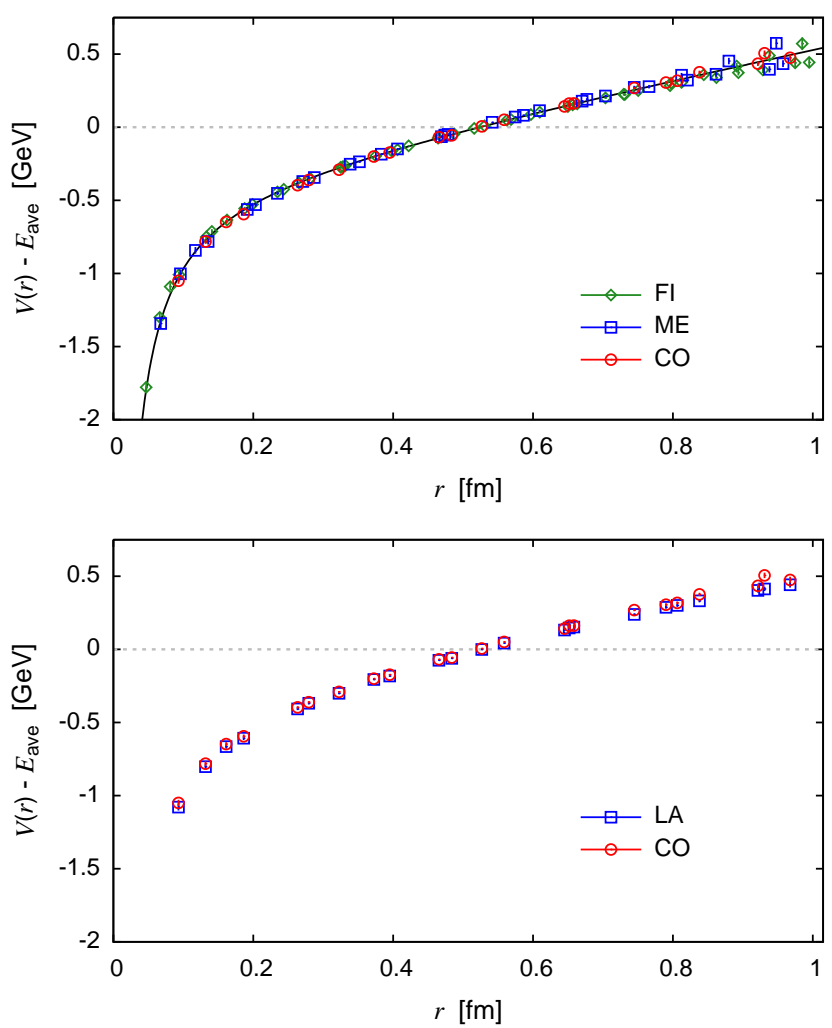

FIG. 11: The lattice spacing dependence (upper) and volume dependence (lower) of spin-independent central potential $V(r)$. For clarity of the figure, the constant energy shift $E_{\text {ave }}$ is not subtracted. In the upper panel, a solid curve shows the fitting results of the Cornell potential form on the data points calculated on the FI ensembles.

Moreover we find the spin-independent central potential determined from our proposed method can maintain the rotational symmetry accurately.

It is also worth noting that no adjustment parameter is added for showing a good scaling of the interquark potential calculated at various $\beta$. This fact is contrast with the case of the static $Q \bar{Q}$ potential given by Wilson loops. For the Wilson loop results, the constant self-energy contributions of infinitely heavy (static) color sources, which will diverge in the continuum limit, must be subtracted to demonstrate the scaling behavior.

The lower panel of Fig. 11 shows no visible finite volume effect on the spin-independent central potential $V(r)$ calculated at charm quark mass at least in the region of $r \lesssim 1 \mathrm{fm}$. This observation is simply due to the fact that the $S$-wave BS wave function at charm quark mass safely fits into even the smaller lattice volume ( $L a \approx 2.2 \mathrm{fm}$ ).

We simply adopt the Cornell potential parameterization for fitting the data of $V(r)$ as

$$
V(r)=-\frac{A}{r}+\sigma r+V_{0}
$$

with the Coulombic coefficient $A$, the string tension $\sigma$, and a constant $V_{0}$. The Cornell potential parameteriza- 
TABLE V: Summary of the Cornell potential parameters $\left(A, \sqrt{\sigma}\right.$ and unsubtracted $\left.V_{0}\right)$, a ratio of $A / \sigma$ and the Sommer parameter $r_{0}$, calculated on all four ensembles.

\begin{tabular}{clllll}
\hline \hline Label & \multicolumn{1}{c}{$A$} & \multicolumn{1}{c}{$\sqrt{\sigma}[\mathrm{GeV}]$} & \multicolumn{1}{c}{$V_{0}-E_{\text {ave }}[\mathrm{GeV}]$} & \multicolumn{1}{c}{$A / \sigma\left[\mathrm{GeV}^{-2}\right]$} & $r_{0}[\mathrm{rm}]$ \\
\hline FI & $0.347(10)(28)(27)(15)$ & $0.439(7)(7)(12)(1)$ & $-0.381(15)(25)(37)(2)$ & $1.804(74)(207)(238)(66)$ & $0.512(8)(3)(8)(4)$ \\
ME & $0.390(13)(36)(25)(0)$ & $0.438(8)(5)(5)(3)$ & $-0.356(19)(26)(21)(7)$ & $2.036(101)(239)(175)(27)$ & $0.505(10)(3)(1)(3)$ \\
CO & $0.382(10)(20)(10)(2)$ & $0.441(6)(5)(4)(3)$ & $-0.370(14)(26)(12)(5)$ & $1.966(76)(132)(86)(15)$ & $0.504(7)(2)(2)(4)$ \\
LA & $0.442(11)(21)(27)(8)$ & $0.428(6)(11)(6)(5)$ & $-0.324(12)(29)(24)(7)$ & $2.418(81)(175)(578)(9)$ & $0.507(8)(13)(2)(8)$ \\
\hline \hline
\end{tabular}

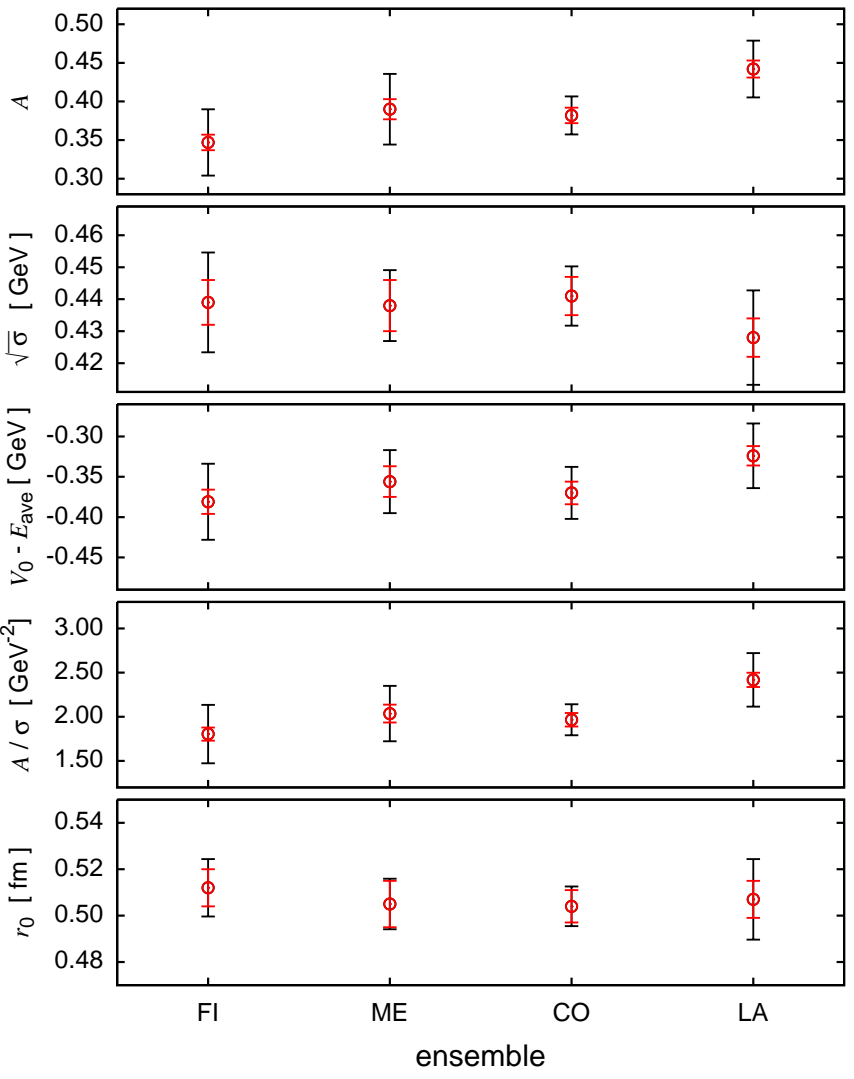

FIG. 12: Summary of the Cornell potential parameters $(A$, $\sqrt{\sigma}$ and $\left.V_{0}\right)$, a ratio of $A / \sigma$ and the Sommer scale $r_{0}$ obtained from all four ensembles (FI, ME, CO and LA). The inner error bars indicate the statistical error, The outer error bars display the total errors, where the statistical and all systematic errors are added in quadrature.

tion describes well the spin-independent central potential even at finite quark mass.

Although the charm quark mass region would be beyond the radius of convergence for the systematic $1 / m_{Q}$ expansion, the finite $m_{Q}$ corrections could be encoded into the Cornell potential parameters in this approach. Table $\mathrm{V}$ presents the summary of the Cornell potential parameters. All fits are performed individually for the three directions (on-axis, off-axis I and II) over the range $0.19 \mathrm{fm} \lesssim r \lesssim 0.84 \mathrm{fm}$. We minimize the $\chi^{2} /$ d.o.f taking into account the covariance matrix.

In Table V, all quoted values of the Cornell potential parameters are obtained by taking an average over the three directions. The first errors are statistical ones. For the second errors, we estimate uncertainties of the choice of the data from the three direction and take the maximal difference from the average among the results of all three directions. Therefore the second errors are associated with the violation of the rotational symmetry. The third and fourth ones are systematic uncertainties originating from the choice of minimum values $\left(t_{\min }\right.$ and $\left.r_{\min }\right)$ of the temporal and spatial windows used in fitting procedures, respectively.

In addition, we estimate a ratio of $A / \sigma$ and the Sommer parameter $r_{0}$, which are also included in Table V. The former is a quantity independent of the definition of the quark mass. In other words, it is simply related to a gross shape of the spin-independent central potential. The later is a well-known phenomenological quantity defined by

$$
r_{0}^{2}=\left.\frac{d V(r)}{d r}\right|_{r=r_{0}}=1.65 .
$$

Thus, $r_{0}$ can be evaluated by the Cornell potential parameters as

$$
r_{0}=\sqrt{\frac{1.65-A}{\sigma}} .
$$

Here we give a few technical remarks on the systematic uncertainties. The value of the string tension $\sigma$ is determined by the long-range behavior of the potential. However, the linear part in the Cornell potential parameterization is dominated in the region where we have data points. Thus, the resulting value of $\sigma$ is relatively insensitive to the choice of the fitting window $\left(r_{\min }, r_{\max }\right)$ and also the choice of the data set with respect to the direction, compared to the Coulombic coefficient $A$. A weak dependence of the latter suggests that a violation of the rotational symmetry is found to be small in the long-range part of the $Q \bar{Q}$ potential. On the other hand, as we described above, the resulting value of $A$ highly depends on the choice of the direction in the fitting procedure. Therefore, there is a large systematic uncertainty associated with the rotational symmetry breaking. This indicates that the short-range part of the $Q \bar{Q}$ potential is not yet fully improved by reducing spatial discretization errors in the discrete Laplacian operator as we proposed in subsection IV B.

The fourth errors tabulated in Table $\mathrm{V}$ are evaluated from uncertainties due to the choice of time window in 
the averaging process over the time slice. These are the smallest errors among the other errors including the statistical one. This is attributed to the fact that we have taken a weighted average of data points in the very wide range of time slices as was discussed in subsection IV C. This particularly contrasts with the conventional approach to calculate the static $Q \bar{Q}$ potential by Wilsonloops or Polyakov lines, where the largest systematic uncertainty is due to the selection of their temporal length.

Fig. 12 displays the Cornell potential parameters $(A$, $\left.\sqrt{\sigma}, V_{0}\right)$, a ratio of $A / \sigma$ and the Sommer scale $r_{0}$, obtained from all four ensembles (FI, ME, CO and LA), for comparison. The inner and outer error bars are the statistical and total errors. The total errors are given by the sum of statistical and systematic errors in quadrature. The resulting Cornell potential parameters calculated at various $\beta$ are consistent within their errors (See results of the FI, ME and $\mathrm{CO}$ ensembles). On the other hand, although the results of the $\mathrm{CO}$ and LA ensembles are consistent within two standard deviations, there appears to be a mild volume dependence on every parameter.

It is worth mentioning here that $r_{0}$ is determined with high accuracy and has no obvious dependence of the lattice spacings and volumes. Then $r_{0}$ agrees well with the input number of $r_{0}=0.5 \mathrm{fm}$ within errors. This is attributed to the fact that the interquark potential at the range, where $V(r)-E_{\text {ave }} \approx 0$, is most precisely determined in the BS amplitude method, while $r_{0}$ is accidentally close to such region.

\section{F. Spin-Spin potential}

We determine the spin-spin potential within the BS amplitude method, through Eq. (11), similar to the spinindependent central potential $V(r)$. Fig. 13 displays the spin-spin potential $V_{\mathrm{S}}(r)$ calculated from the $Q \bar{Q}$ BS wave function. First the resulting potential is quickly dumped at large distances and exhibits a repulsive interaction with a finite range of $r \lesssim 0.6 \mathrm{fm}$. This is different from a short-range $\delta$-function potential based on one-gluon exchange like the Fermi-Breit interaction of QED. Second repulsive interaction is required by the charmonium spectroscopy, where the higher spin state in hyperfine multiplets receives heavier mass. It should be reminded that the Wilson loop approach fails to reproduce the correct behavior of the spin-spin interaction even in the bottom sector. The leading-order contribution of the spin-spin potential classified in PNRQCD gives rise to a short-range attractive interaction, which yields wrong mass ordering among hyperfine multiplets [27].

As shown in the upper panel of Fig. 13, the discretization artifacts are visible on the spin-spin potential at the short distances, where the scaling behavior is violated. This contrasts to the spin-independent central potential, where a good scaling behavior is observed even at the short distances. However, this observation is consistent with the fact that the hyperfine splitting energies ex-
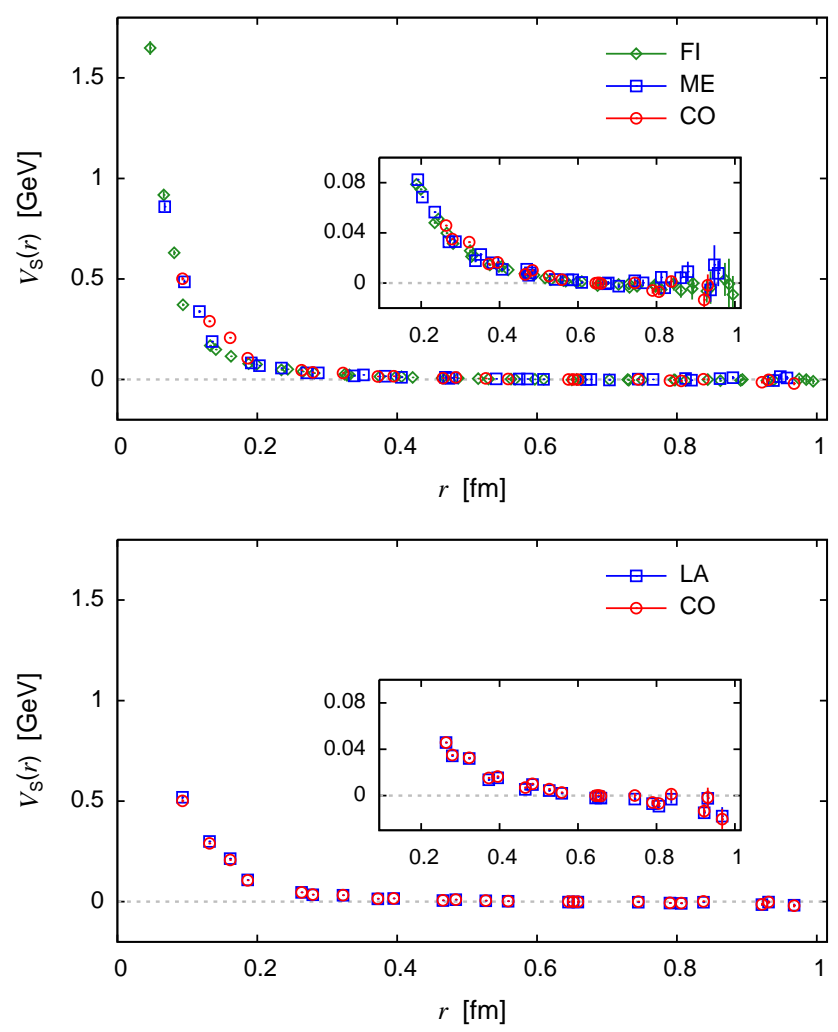

FIG. 13: The lattice spacing dependence (upper) and volume dependence (lower) of the spin-spin potential $V_{\mathrm{S}}(r)$. The insets show magnified views in the region of $r \gtrsim 0.1 \mathrm{fm}$.

hibit a slight, but systematic dependence of the lattice spacing (See Fig. 3). In order to determine the spinspin potential keeping systematics under control, we will need simulations on finer lattices, or alternatively perform nonperturbative tuning of the RHQ parameters and further improvement of the discrete Laplacian operator.

On the other hand, as for the finite volume effect, there is no significant difference between the spin-spin potentials calculated from two different physical volumes $(\mathrm{CO}$ and LA) as shown in the lower panel of Fig. 13. This is consistent with the fact that the spin-spin potential $V_{\mathrm{S}}(r)$ is measured as the short-range potential and the BS wave function at short distances is insensitive to the spatial extent.

\section{HEAVY QUARK MASS LIMIT OF INTERQUARK POTENTIAL}

In this section, we discuss an asymptotic behavior of both the spin-independent central and spin-spin potentials in the heavy quark mass limit $m_{Q} \rightarrow \infty$. We will first show that the spin-independent central potential in the $m_{Q} \rightarrow \infty$ limit is fairly consistent with the conventional one obtained from Wilson-loops or Polyakov lines. For this purpose, we examine the quark mass dependence 
TABLE VI: Summary of the RHQ parameters $\left(\nu, r_{s}, c_{B}\right.$ and $\left.c_{E}\right)$ and spin-averaged masses of the $1 S$ heavy quarkonium state, used in the simulation with the FI ensembles toward the infinitely heavy quark limit.

\begin{tabular}{lcccccl}
\hline \hline & $\kappa_{Q}$ & $\nu$ & $r_{s}$ & $c_{B}$ & $c_{E}$ & $M_{\text {ave }}[\mathrm{GeV}]$ \\
\hline charm & 0.11727 & 1.029 & 1.131 & 1.700 & 1.562 & $3.0676(20)$ \\
& 0.11198 & 1.041 & 1.165 & 1.749 & 1.581 & $3.9612(16)$ \\
& 0.10377 & 1.066 & 1.230 & 1.842 & 1.619 & $5.1925(13)$ \\
bottom & 0.09004 & 1.124 & 1.364 & 2.033 & 1.708 & $7.2466(11)$ \\
& 0.07619 & 1.211 & 1.543 & 2.388 & 1.839 & $9.4462(9)$ \\
& 0.05759 & 1.402 & 1.906 & 2.807 & 2.127 & $12.8013(8)$ \\
\hline \hline
\end{tabular}

of the potentials near the infinitely heavy quark mass as much as possible.

To avoid further discretization errors induced by heavier quark masses, we choose the finest lattice spacing ensembles (FI) and perform additional simulations with extra five hopping parameters, which corresponds to the heavier quark masses than the charm quark sector. The inverse of lattice spacing on the FI ensembles is about $4.2 \mathrm{GeV}$, which is closet to the bottom mass. Therefore, we choose our hopping parameters covering a wide mass range from the charm to beyond the bottom region toward the heavy quark limit.

At the second heaviest quark mass $\left(\kappa_{Q}=0.07619\right)$, we obtain the spin-averaged $1 S$-heavy quarkonium mass as $M_{\text {ave }}=9.4462(9) \mathrm{GeV}$, which is close to the experimental one of the bottomonium. Thus, $\kappa_{Q}=0.07619$ is reserved for the bottom quark mass. It is worth mentioning that the hyperfine splitting energy calculated at the bottom quark mass in our simulations reproduces only $40 \%$ of the experimental value [53]. At each $\kappa_{Q}$, we again use the one-loop perturbation theory to determine five RHQ parameters following Ref. [54]. These RHQ parameters, which are summarized with given values of $\kappa_{Q}$ in Table VI, marginally satisfies the condition of $c_{\text {eff }}^{2}=1$ for the $1 S$ heavy quarkonium states at all five quark masses within errors.

\section{A. BS wave function}

In Fig. 14, we first plot the reduced $Q \bar{Q}$ BS wave functions of the pseudoscalar quarkonium calculated at various quark masses. These wave functions are normalized as to fulfill the condition $\sum \tilde{\phi}^{2}=1$. We again find the isotropic behavior in the BS wave functions even at around the bottom quark mass. The data points calculated from the three directions basically collapse on a single curve. Nothing changes for the vector quarkonium wave function.

The wave function with a heavier quark mass is more localized than the one with a lighter quark mass. Thus, the finite volume effect on the interquark potential becomes not serious at around the bottom quark mass. For the price one has to pay, a number of accessible data

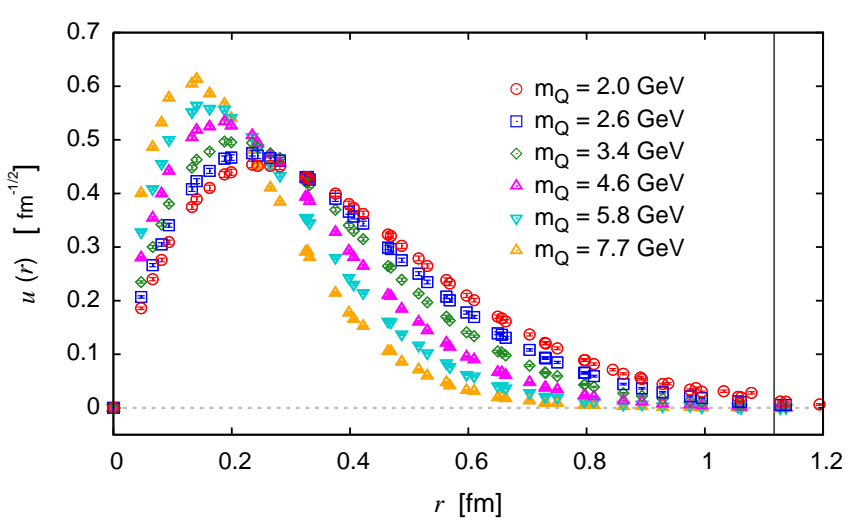

FIG. 14: The reduced $Q \bar{Q}$ BS wave functions of the pseudoscalar quarkonium state calculated using the FI ensembles with six different quark masses covering the range from $2.0 \mathrm{GeV}$ to $7.7 \mathrm{GeV}$, shown as a function of the spatial distance of $r$. A vertical solid line denotes a half of the lattice size $(L a / 2 \approx 1.1 \mathrm{fm})$.

TABLE VII: Results of the quark kinetic mass $m_{Q}$, the Cornell potential parameters $A, \sqrt{\sigma}$, and the ratio $A / \sigma$, calculated on the FI ensembles. Their extrapolated values in the $m_{Q} \rightarrow \infty$ limit using linear and quadratic fit forms are compared with our results given by the Polyakov line correlator and also accurate results calculated with the multilevel algorithm [27].

\begin{tabular}{lcllc}
\hline \hline$\kappa_{Q}$ & $\begin{array}{c}m_{Q} \\
{[\mathrm{GeV}]}\end{array}$ & \multicolumn{1}{c}{$A$} & $\begin{array}{c}\sqrt{\sigma} \\
{[\mathrm{GeV}]}\end{array}$ & $\begin{array}{c}A / \sigma \\
{\left[\mathrm{GeV}^{-2}\right]}\end{array}$ \\
\hline 0.11727 & $2.00(5)$ & $0.323(9)$ & $0.447(6)$ & $1.62(5)$ \\
0.11198 & $2.60(5)$ & $0.297(6)$ & $0.443(5)$ & $1.51(4)$ \\
0.10377 & $3.36(6)$ & $0.288(6)$ & $0.439(5)$ & $1.49(5)$ \\
0.09004 & $4.57(7)$ & $0.279(5)$ & $0.441(5)$ & $1.43(4)$ \\
0.07619 & $5.80(7)$ & $0.277(4)$ & $0.445(5)$ & $1.40(4)$ \\
0.05759 & $7.71(8)$ & $0.277(4)$ & $0.446(5)$ & $1.39(4)$ \\
\hline linear fit & $\infty$ & $0.273(9)$ & $0.454(11)$ & $1.31(9)$ \\
quadratic fit & $\infty$ & $0.285(11)$ & $0.454(12)$ & $1.40(9)$ \\
\hline static $Q \bar{Q}$ (Polyakov lines) & $0.285(11)$ & $0.467(6)$ & $1.31(8)$ \\
static $Q \bar{Q}$ (Ref. $[27])$ & $0.281(5)$ & $0.458(1)$ & $1.34(2)$ \\
\hline \hline
\end{tabular}

points at long distances gradually reduces for heavier quark mass. It is worth reminding that in the BS amplitude method, we cannot access the information of the interquark potential outside of the localized wave function, where the wave function approximately vanishes and a signal-to-noise ratio in $\nabla^{2} \phi_{\Gamma} / \phi_{\Gamma}$ gets worse.

\section{B. Spin-independent interquark potential}

Fig. 15 displays the spin-independent central potential (upper) and spin-spin potential (lower) calculated at several quark masses within the BS amplitude method. In the upper panel of Fig. 15, the constant energy shift $E_{\text {ave }}$ is not subtracted as same in Fig. 11. At first glance, the "Coulomb plus confining potential" are observed over 


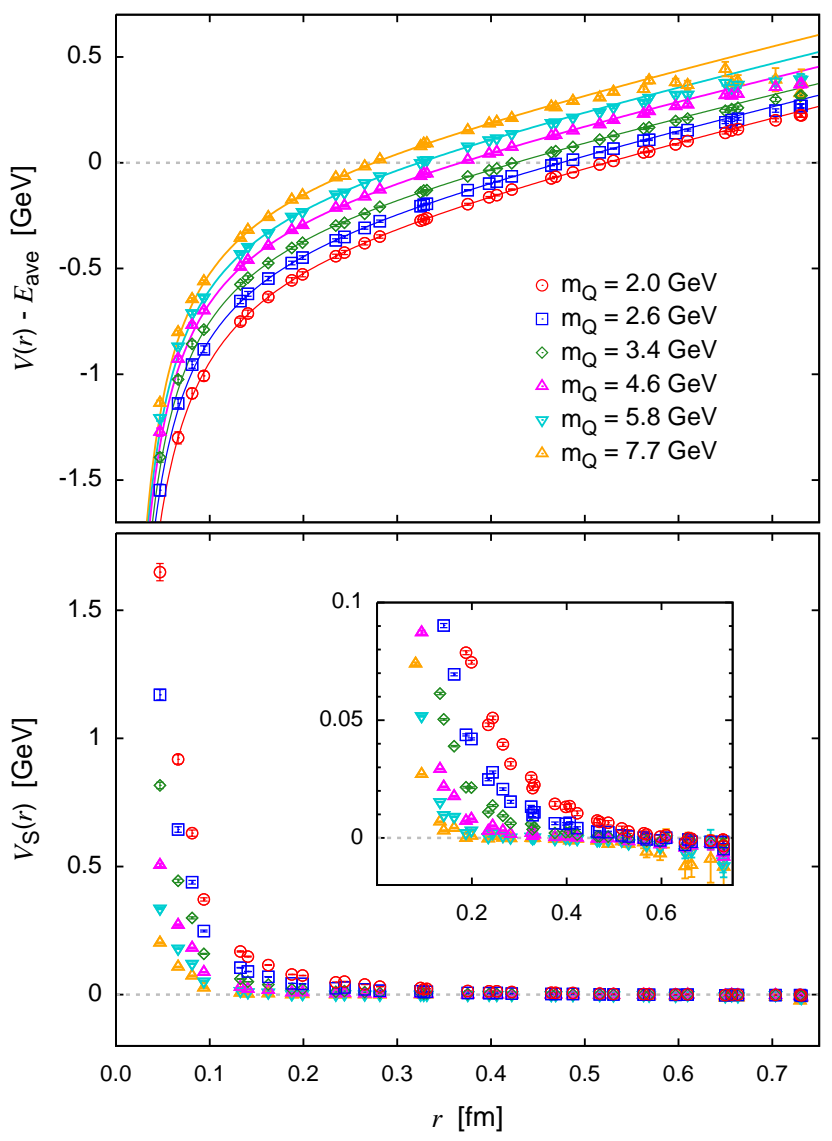

FIG. 15: The spin-independent central (upper) and spin-spin (lower) potential calculated from the $Q \bar{Q}$ BS wave function at finite quark masses covering the range from $2.0 \mathrm{GeV}$ to $7.7 \mathrm{GeV}$. In the upper panel, each curve represents the fitting result of the Cornell potential form given in Eq. (20), and also the constant energy shift $E_{\text {ave }}$ is not subtracted. The inset in the lower panel shows a magnified view in the region of $r \lesssim 0.6 \mathrm{fm}$.

range from the charm to the bottom quark mass. We perform a fit of the potentials calculated at various quark masses to a simple form of the Coulomb plus linear potential, then obtain the Cornell potential parameters, which are summarized in Table. VII. All fits are performed over the range $3 \leq r / a \leq 7 \sqrt{3}$ by correlated $\chi^{2}$ fit. The errors quoted in Table. VII are only statistical uncertainties, which are estimated by the jackknife method.

In Fig. 16, we show the quark-mass dependence of the ratio of $A / \sigma$ (upper), the Coulombic coefficient $A$ (middle) and the squared-string tension $\sqrt{\sigma}$ (lower). We also include values of the static $Q \bar{Q}$ potential calculated from the Polyakov line correlator $P(r, t)$ as reference values in the infinitely heavy quark limit. The static $Q \bar{Q}$ potential are obtained by fitting a plateau of the effective potential $V_{\text {eff }}(r, t)=\ln \{P(r, t) / P(r, t+1)\}$ over range $\left[t_{\text {min }}, t_{\text {mas }}\right]=[7: 10]$. The Cornell potential parameters can be obtained by applying the same fitting procedure used in the case of the BS amplitude method. We ad-

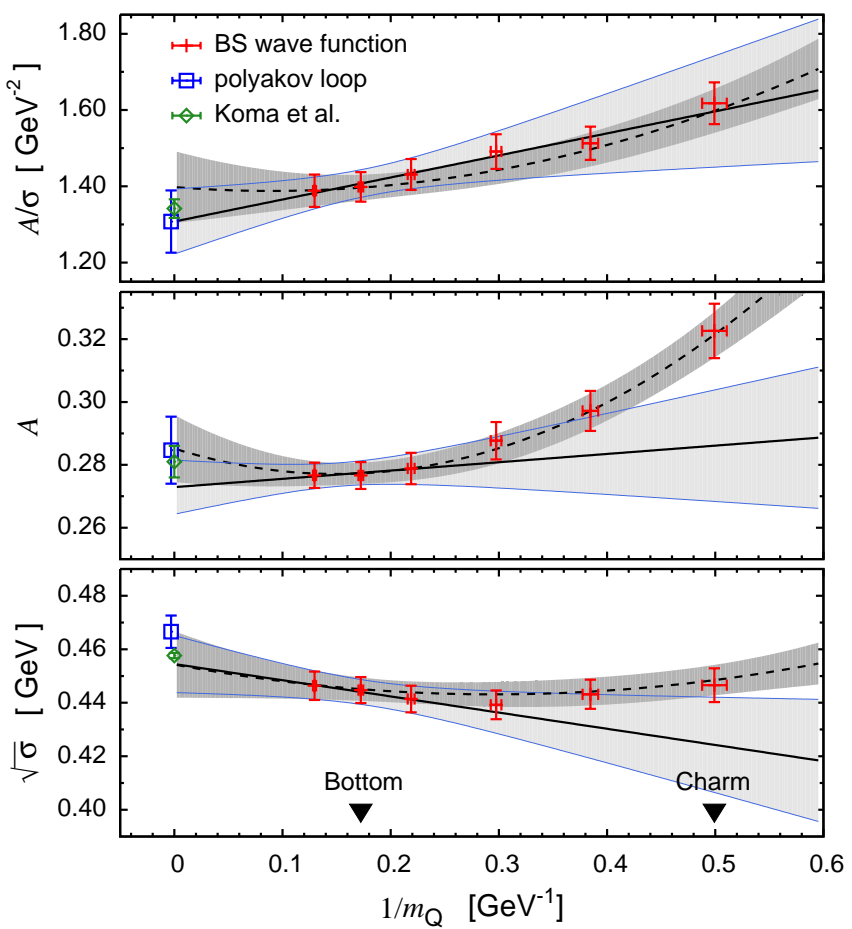

FIG. 16: The quark-mass dependence of $A / \sigma$ (upper), $A$ (middle) and $\sqrt{\sigma}$ (lower), shown as functions of $1 / m_{Q}$. We perform the extrapolation toward the $m_{Q} \rightarrow \infty$ limit of $A / \sigma$, $A$ and $\sigma$ with a simple polynomial function in $1 / m_{Q}$. Solid lines and dashed curves in each panel indicate the fitting results of linear and quadratic forms, respectively. Shaded bands show statistical fitting uncertainties estimated by the jackknife method. The results of the static $Q \bar{Q}$ potential calculated by the Polyakov line correlator and also the Wilson loop using the multilevel algorithm [27] are also included as square and diamond symbols.

ditionally include more accurate results given by Wilson loops using the multilevel algorithm [27].

First, regardless of the definition of $m_{Q}$, the ratio of $A / \sigma$ in the upper panel of Fig. 16 indicates that the interquark potential calculated from the BS wave function smoothly approaches the one obtained from Wilson loops in the infinitely heavy quark limit. The extrapolation toward the $m_{Q} \rightarrow \infty$ limit is consistent with the value obtained from the static $Q \bar{Q}$ potentials. Here, we perform both linear (solid line) and quadratic (dashed curve) fits with respect to $1 / m_{Q}$ to three heaviest points and all of six data points, respectively. All fits take into account the correlations among the different mass data in correlated $\chi^{2}$ fit. Shaded bands appeared in Fig. 16 indicate statistical errors, which are estimated by the jackknife method.

Second, if we pay attention to the quark-mass dependence of each of the Cornell potential parameters separately, we observe that the Coulombic parameter $A$ depends on the quark mass significantly, while there is no appreciable dependence of the quark mass on the string 

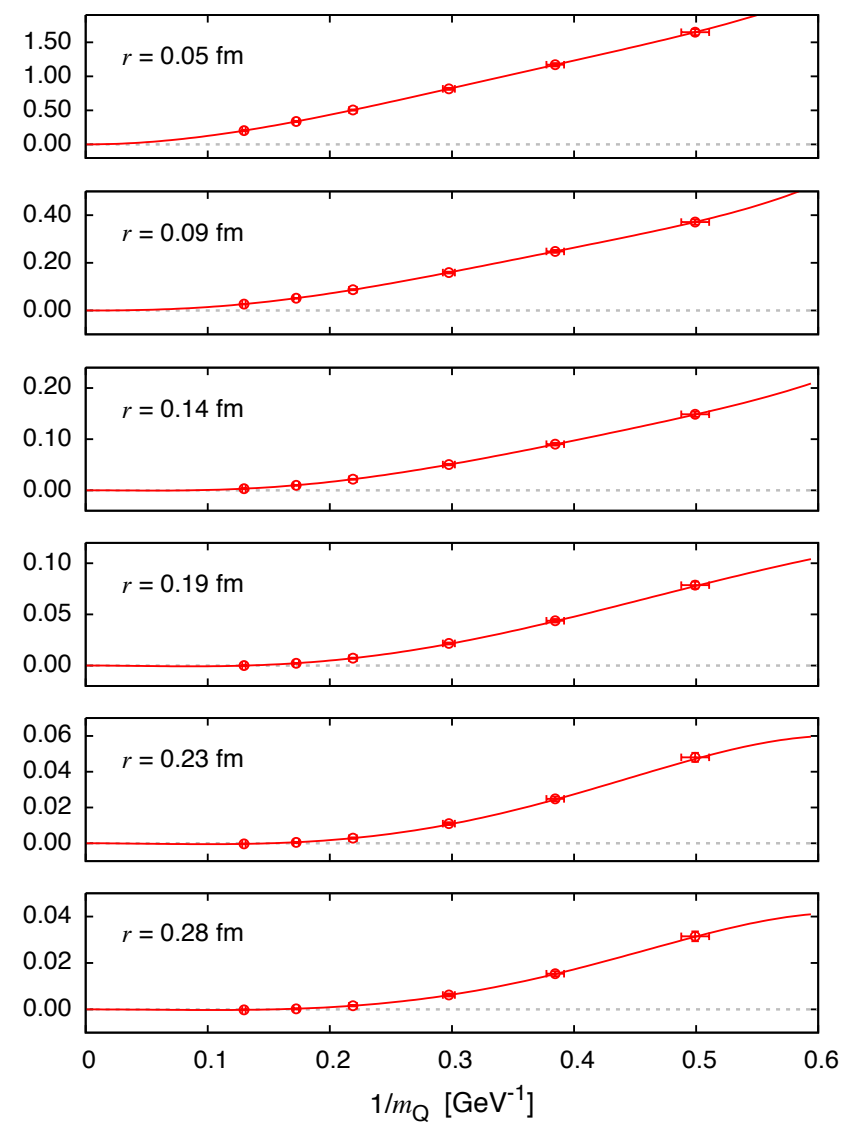

FIG. 17: The quark mass dependences of the spin-spin potential $V_{\mathrm{S}}(r)$ at fixed $r$ as functions of $1 / m_{m_{Q}}$. The selected values of $r$ are indicated in each panel. The vertical axis is plotted in units of $\mathrm{GeV}$. Solid curves correspond to fitting results of the polynomial forms given in Eq. (23).

tension (see the middle and lower panels in Fig. 16). The finite $m_{Q}$ corrections seem to appear mainly in the shortrange part of the potential characterized by the Coulombic coefficient $A$. At the charm quark mass, higher order corrections, at least the $\mathcal{O}\left(1 / m_{Q}^{2}\right)$ corrections, could be quite important to describe the spin-independent central potential.

We finally evaluate the values of $A$ and $\sqrt{\sigma}$ in the infinitely heavy quark limit by both quadratic and linear fits as shown in Fig. 16, and also the results are summarized in Table VII. Extrapolated values in the $m_{Q} \rightarrow \infty$ limit are consistent with those of the static $Q \bar{Q}$ potentials. We stress that our proposed method for determining the interquark potential with the proper quark mass given in Eq. (13) is responsible for the quark-mass dependence observed here.

\section{Spin-spin potential}

The quark-mass dependence of the spin-spin potential is more pronounced in contrast to the spin-independent central potential (see the lower panel of Fig. 15). As the quark mass increases, a finite range of the spin-spin interaction becomes narrower, and then the potential seems to approach the $\delta$-function potential, which would be induced by one-gluon exchange. We may expect that the spin-spin potential obtained in the BS amplitude method has a correct behavior toward the $m_{Q} \rightarrow \infty$ limit.

The spin-dependent potential in pNRQCD appears as the $1 / m_{Q}$ corrections to the static $Q \bar{Q}$ potential. However there is a huge gap between our spin-spin potential at finite quark mass and one determined at $\mathcal{O}\left(1 / m_{Q}^{2}\right)$ within the systematic $1 / m_{Q}$ expansion approach $[27,28]$. The former exhibits the short-range repulsive interaction, while the latter is similarly short-ranged, but turns out to be slight attractive interaction near the origin.

To resolve the issue of the qualitative difference between two methods, we try to read off the corresponding leading and also higher order corrections in the $1 / m_{Q}$ expansion from our spin-spin potential, where all orders in the $1 / m_{Q}$ expansion are supposed to be nonperturbatively encoded. We thus try to parametrize the spin-spin potential calculated with the finite quark mass $m_{Q}$ in guidance of pNRQCD $^{1}$ as

$$
V_{\mathrm{S}}\left(m_{Q}, r\right)=\frac{1}{m_{Q}^{2}}\left(V_{\mathrm{S}}^{(2)}(r)+\frac{1}{m_{Q}} V_{\mathrm{S}}^{(3)}(r)+\cdots\right) .
$$

In Refs. [27, 28], the leading order contribution of $V_{\mathrm{S}}^{(2)}(r)$ is precisely determined within the Wilson loop formalism using the multilevel algorithm. As was already mentioned, their spin-spin potential exhibits slight attractive interaction near the origin.

In Fig. 17 we plot the spin-spin potential at fixed $r$ as a function of $1 / m_{Q}$. At every $r$, we have carried out correlated $\chi^{2}$ fits on all six data displayed in Fig. 17 by using a polynomial form of $1 / m_{Q}$, according to Eq. (23). The $m$-th coefficient of the polynomial expansion with respect to $1 / m_{Q}$ can be identified as the potential value of $V_{\mathrm{S}}^{(m+1)}(r)$ at given $r$, corresponding to the correction term at $\mathcal{O}\left(1 / m_{Q}^{m+1}\right)^{2}$. The fit results are also displayed as solid curves in Fig. 17. The stability of the fit results has been tested against either the number of fitted data

1 Odd powers of $1 / m_{Q}$ could appear in the case of non-abelian gauge theory [56].

2 The same analysis, in principle, can be applied to the spinindependent central potential. The leading order potential $V^{(0)}(r)$, which corresponds to the $Q \bar{Q}$ potential in the $m_{Q} \rightarrow \infty$ limit, was obtained in this procedure. We have confirmed that $V^{(0)}(r)$ obtained in this analysis is fairly consistent with the static $Q \bar{Q}$ potential calculated from the Polyakov line correlator. However, the spin-independent central potential involves the self energy of a quark and anti-quark pair, which is proportional to 

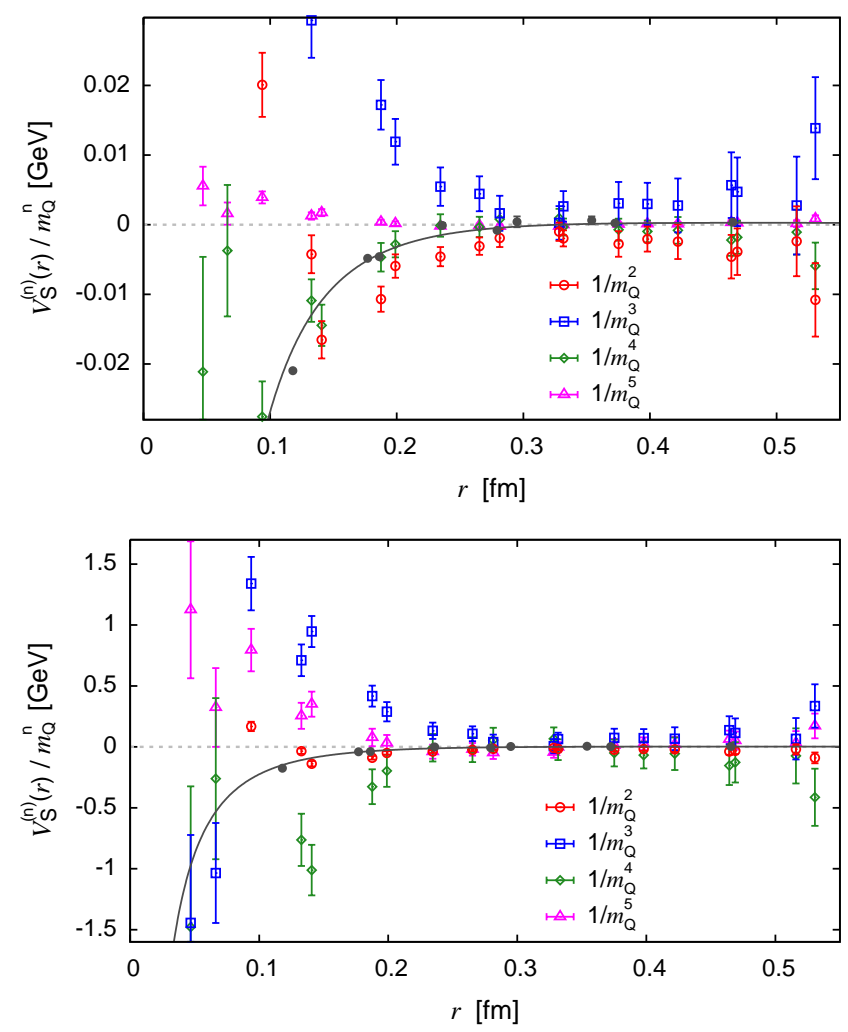

FIG. 18: The $r$-dependence of the inverse quark mass corrections $V_{\mathrm{S}}^{(n)}(r) / m_{Q}^{n}$ on the spin-spin potential at the bottom (upper) and charm (lower) quark masses. Filled circles correspond to the spin-spin potential at $\mathcal{O}\left(1 / m_{Q}^{2}\right)$ calculated within the Wilson loop formalism, together with their fit results (solid curves) [27, 28].

points or the number of the polynomial terms. We find that the polynomial terms up to the $\mathcal{O}\left(1 / m_{Q}^{5}\right)$ term are necessary to describe the quark mass dependence of the spin-spin potential, covering a whole range of $2.0 \mathrm{GeV}$ $\leq m_{Q} \leq 7.7 \mathrm{GeV}$, due to the slow convergence of the $1 / m_{Q}$ expansion in the vicinity of the charm sector. Our choice of the maximum polynomial term of $\mathcal{O}\left(1 / m_{Q}^{5}\right)$ in the fitting form as Eq. (23) certainly yields acceptable values of $\chi^{2} /$ d.o.f and confidence level.

In Fig. 18, we compile all results of $V_{\mathrm{S}}^{(n)}(r)$ (up to $n=5$ ), scaling with powers of $1 / m_{Q}^{n}$, in order to analyze the convergence behavior of the $1 / m_{Q}$ expansion

$m_{Q}$ as

$V\left(m_{Q}, r\right)=$ constant $\times m_{Q}+V^{(0)}(r)+\frac{1}{m_{Q}} V_{\mathrm{S}}^{(1)}(r)+\cdots$.

The presence of a term of $\mathcal{O}\left(m_{Q}\right)$ in addition to the polynomial function of $1 / m_{Q}$ makes the fit relatively unstable, compared to the case of the spin-spin potential. Unfortunately, we did not observe the stability of the fit results even for the leading order correction of $\mathcal{O}\left(1 / m_{Q}\right)$ within the current statistics. at both the bottom (upper) and charm (lower) quark masses. As shown in the upper panel of Fig. 18, the $\mathcal{O}\left(1 / m_{Q}^{2}\right)$ contribution (open circles) to the total spinspin potential exhibits an exponentially screened at the long distances and attractive interaction in the intermediate region $(0.1 \mathrm{fm} \lesssim r \lesssim 0.3 \mathrm{fm})$. Surprisingly, the $\mathcal{O}\left(1 / m_{Q}^{3}\right)$ contribution (open squares) is the largest contribution, and ensures the short-range repulsive interaction of the total spin-spin potential.

Here, we remark on the short-range behavior found in the $\mathcal{O}\left(1 / m_{Q}^{2}\right)$ contribution near the origin. At the short distances $(r \lesssim 0.1 \mathrm{fm})$, the sign of the spin-spin potential changes from negative to positive. We will later explain the reason why we do not take it seriously and then let us focus on results obtained in the region of $r \gtrsim 0.1 \mathrm{fm}$.

The solid curve represents the fit curve on the data points (filled circles) taken from Ref. [27, 28], scaled by $1 / m_{Q}^{2}$ with the bottom quark mass, $m_{Q}=5.80(7) \mathrm{GeV}$. The size of the attraction found in the $\mathcal{O}\left(1 / \mathrm{m}_{Q}^{2}\right)$ contribution is almost the same order of magnitude as that of the spin-spin potential determined within the Wilson loop formalism [27, 28].

At this point, we may have a hint to fill out a gap between our results of the spin-spin potential calculated in the BS amplitude method and one calculated at $\mathcal{O}\left(1 / m_{Q}^{2}\right)$ within the $1 / m_{Q}$ expansion scheme. According our analysis, the next-to-leading order contribution of $\mathcal{O}\left(1 / m_{Q}^{3}\right)$ is not negligible, rather a dominant contribution in the full spin-spin potential. In other words, the issue of the spin-spin potential in the $1 / m_{Q}$ expansion approach within the Wilson loop formalism would be cured by the next-leading-order contribution of $\mathcal{O}\left(1 / m_{Q}^{3}\right)$. Furthermore, although the sizes of $\mathcal{O}\left(1 / m_{Q}^{2}\right)$ and $\mathcal{O}\left(1 / m_{Q}^{3}\right)$ contributions are inverted in the sense of the systematic $1 / m_{Q}$ expansion, the higher order contributions are certainly smaller than a sum of the two lowest contributions at the bottom quark mass. Therefore, our analysis suggests that the $1 / m_{Q}$ expansion scheme may have the convergence behavior up to the bottom sector.

It is, however, not the case for the charm sector. In the lower panel of Fig. 18, we plot the similar figure which are scaled with the charm quark mass $m_{Q}=2.00(5)$ $\mathrm{GeV}$ in the scaling factor $1 / m_{Q}^{n}$. The largest contribution is still the $\mathcal{O}\left(1 / m_{Q}^{3}\right)$ contribution, while the size of higher order contributions becomes comparable to that of the $\mathcal{O}\left(1 / m_{Q}^{3}\right)$ contribution. Obviously the higher order corrections are much important rather than the leading order correction of $\mathcal{O}\left(1 / m_{Q}^{2}\right)$ at the charm quark mass. Nevertheless, the signs of the higher order contributions clearly alternate between positive and negative. Remark that the full spin-spin potential is certainly repulsive in a whole range of $r$ measured here. The higher order contributions of $\mathcal{O}\left(1 / m_{Q}^{4}\right)$ and $\mathcal{O}\left(1 / m_{Q}^{5}\right)$ are almost canceled with each other and then the $\mathcal{O}\left(1 / m_{Q}^{3}\right)$ contribution approximately represents a whole nature of repulsion of the full spin-spin potential.

These observations may indicate that the $1 / m_{Q}$ ex- 
pansion is no longer converged in the charm quark mass region. In this sense, the new determination of the interquark potential at finite quark mass within the BS amplitude method is a powerful tool for exploring the charmonium system. We can compute theoretical inputs for modeling the reliable interquark potential from first principles QCD, and then provide new and valuable information to especially the spin-dependent potentials including the tensor and spin-orbit potentials in the quark potential models.

Finally, we make a comment on the peculiar behavior found in the $\mathcal{O}\left(1 / m_{Q}^{2}\right)$ contribution near the origin. We first recall that a residual discretization error that may not be removed in the RHQ action is of order $\mathcal{O}\left((a p)^{2}\left(a m_{Q}\right)\right)$. The inverse of lattice spacing for the FI ensembles used here is about $4.2 \mathrm{GeV}$, which is not quite higher than the bottom quark mass, rather lower than our three heaviest quark masses $\left(m_{Q}=4.57(7)\right.$, $5.80(7)$ and $7.71(8) \mathrm{GeV}$ ) in this study. Therefore, our data set of the interquark potentials in principle suffers from the the residual discretization error, which may not be serious in simulations at the charm quark mass. In the analysis discussed here, the data obtained at heavier quark masses is obviously important. Therefore, the final results, which highly relies on the heavy quark mass extrapolation, should receive some influence of the residual discretization error, which is not negligible in the short distance region of $r \lesssim 1 / p \lesssim a\left(a m_{Q}\right)^{1 / 2} \sim 0.1 \mathrm{fm}$. Therefore, in above discussions, we simply disregard the shortrange behavior that we should not take seriously.

\section{SUMMARY}

We have proposed the new method to determine the interquark potential at finite quark mass in lattice QCD. The $Q \bar{Q}$ potential is defined through the equal-time $Q \bar{Q}$ Bethe-Salpeter wave function and also the quark kinetic mass is self-consistently determined on the same footing. The proper definition of the quark mass is essential for the application of the BS amplitude method to the $Q \bar{Q}$ system. The spin-independent and dependent parts of interquark potential together with the quark kinetic mass can be calculated with a single set of four-point correlation functions.

We have demonstrated the feasibility of our new proposal by using quenched lattice QCD simulations. In order to study several systematic uncertainties on the interquark potential, our simulations were performed on several gauge ensembles generated in the quenched approximation at three different lattice spacings, $a \approx$ 0.093, 0.068 and $0.047 \mathrm{fm}$, and two different physical volumes, $L a \approx 2.2$ and $3.0 \mathrm{fm}$. The heavy quark propagators were computed using the RHQ action with the coefficients determined by one-loop perturbative calculations. The hopping parameter was chosen to reproduce the experimental spin-averaged mass of the $1 S$ charmonium states.
In the BS amplitude method, there is a room for optimizing the differential operator since the discrete Laplacian operator is itself built in the definition of the interquark potential. Through the simulations carried out at three different lattice spacings, we first conclude that the discrete Laplacian operator in the discrete polar coordinates is more suitable than the naive one defined in the Cartesian coordinates to reduce the discretization artifacts on the short-range behavior of the interquark potential. The resultant spin-independent central potential in quenched lattice QCD exhibits the linearly rising potential at large distances and the Coulomb-like potential at short distances. All results calculated at three different lattice spacings nicely collapse on a single curve. In this sense, the rotational symmetry is effectively recovered in the spin-independent central potential calculated in the BS amplitude method. We also confirm, through simulations on two different physical volumes, that the finite volume effect on the interquark potential is negligible if the BS wave function safely fits into the lattice volume used for the simulation.

We have additionally examined the quark mass dependence of the interquark potential over a wide mass range from the charm to beyond the bottom toward the infinitely heavy quark limit, using the finest lattice spacing ensembles (the inverse of lattice spacing is $\left.1 / a_{48} \approx 4.2 \mathrm{GeV}\right)$. We then demonstrated that the spinindependent central potential in the $m_{Q} \rightarrow \infty$ limit is connected to the static interquark obtained from Wilsonloops and Polyakov lines, and find that the $\mathcal{O}\left(1 / m_{Q}^{2}\right)$ correction should be non-negligible on the short-range part of the spin-independent central potential at around the charm quark mass.

The spin-spin potential at finite quark mass in quenched lattice QCD provides not pointlike, but finiterange repulsive interaction. The spin-spin potential determined in the new method potentially accounts for all orders of $1 / m_{Q}$ corrections, and also shows the qualitative difference from the slightly attractive spin-spin potential measured at $\mathcal{O}\left(1 / m_{Q}^{2}\right)$ in pNRQCD. The repulsive feature of the spin-spin interaction is phenomenologically required by the observed mass-ordering found in hyperfine multiplets. The issue on the spin-spin potential determined in the $1 / m_{Q}$ expansion approach may be resolved by what we found in a detailed study of quark mass dependence on the spin-spin potential calculated by the BS amplitude method.

We read off from our spin-spin potential, which may encode all orders of the $1 / m_{Q}$ expansion, that the corresponding $\mathcal{O}\left(1 / m_{Q}^{2}\right)$ correction exhibits the slight attraction and then barely agrees with the Wilson-loop results. Furthermore, the most important contribution to the spin-spin potential should be the $\mathcal{O}\left(1 / m_{Q}^{3}\right)$ correction, which is responsible for the repulsive feature of the total spin-spin potential, rather than the $\mathcal{O}\left(1 / m_{Q}^{2}\right)$ correction even at the bottom quark mass.

We finally conclude that both the spin-independent central and spin-spin potentials calculated at finite quark 
mass in the BS amplitude method can reproduce known results calculated within the Wilson loop formalism in the infinitely heavy quark limit. Apparently the new method proposed in this paper has the advantage of determining the proper $Q \bar{Q}$ potential in not only the bottom sectors, but also the charm sector.

From the viewpoint of phenomenology, greater knowledge of the $r$-dependence of the spin-dependent potentials paves way for making more accurate theoretical predictions about the higher-mass quarkonium states. Indeed, the $r$-dependence of the spin-spin potential calculated from first principles of QCD is significantly different from a repulsive $\delta$-function potential of the Fermi-Breit interaction, which is widely adopted in quark potential models.

In this sense, a full set of the reliable spin-dependent potentials derived from lattice QCD can provide new and valuable information to the quark potential models. We plan to develop our method to determine all spindependent potentials including the tensor and spin-orbit forces. The tensor one is especially required to quantify the size of a mixing between $1 S$ and $1 D$ wave functions, that is assumed to be negligible in the vector quarkonium states in our current analysis. Such planning is now under way.

\section{Acknowledgments}

It is a pleasure to acknowledge helpful suggestions with T. Hatsuda, and to thank H. Iida and Y. Ikeda for fruitful discussions. S.S. also thanks to Y. Sumino for discussion on the next-to-leading order term of the spinspin potential in the $1 / m_{Q}$ expansion. This work is supported by JSPS Grants-in-Aid for Scientific Research (No. 23540284). T.K. is partially supported by JSPS Strategic Young Researcher Overseas Visits Program for Accelerating Brain Circulation (No. R2411).
[1] F. Close, An introduction to quarks and partons, Academic Press/london 1979, 481p.

[2] E. Eichten, K. Gottfried, T. Kinoshita, J. B. Kogut, K. Lane, et al., Phys. Rev. Lett. 34, 369 (1975).

[3] E. Eichten, K. Gottfried, T. Kinoshita, K. Lane, and T.M. Yan, Phys. Rev. D17, 3090 (1978).

[4] E. Eichten, K. Gottfried, T. Kinoshita, K. Lane, and T.M. Yan, Phys. Rev. D21, 203 (1980).

[5] J. L. Richardson, Phys.Lett. B82, 272 (1979).

[6] T. Barnes, S. Godfrey, and E. Swanson, Phys. Rev. D72, 054026 (2005), hep-ph/0505002.

[7] E. Eichten and F. Feinberg, Phys. Rev. D23, 2724 (1981).

[8] S. Godfrey and N. Isgur, Phys. Rev. D32, 189 (1985).

[9] S. Godfrey and S. L. Olsen, Ann.Rev.Nucl.Part.Sci. 58, 51 (2008), hep-ph/0801.3867.

[10] G. S. Bali, Phys. Rept. 343, 1 (2001), hep-ph/0001312.

[11] G. Bali and K. Schilling, Phys. Rev. D46, 2636 (1992).

[12] K. Schilling and G. Bali, Int. J. Mod. Phys. C4, 1167 (1993), hep-lat/9308014.

[13] G. S. Bali and K. Schilling, Phys. Rev. D47, 661 (1993), hep-lat/9208028.

[14] S. Booth et al. (UKQCD Collaboration), Phys. Lett. B294, 385 (1992), hep-lat/9209008.

[15] U. Glassner et al. (TXL Collaboration), Phys. Lett. B383, 98 (1996), hep-lat/9604014.

[16] N. Brambilla, A. Pineda, J. Soto, and A. Vairo, Rev.Mod. Phys. 77, 1423 (2005), hep-ph/0410047.

[17] P. de Forcrand and J. D. Stack, Phys. Rev. Lett. 55, 1254 (1985).

[18] C. Michael and P. E. Rakow, Nucl. Phys. B256, 640 (1985).

[19] C. Michael, Phys. Rev. Lett. 56, 1219 (1986).

[20] A. Huntley and C. Michael, Nucl. Phys. B286, 211 (1987).

[21] M. Campostrini, K. Moriarty, and C. Rebbi, Phys. Rev. Lett. 57, 44 (1986).

[22] M. Campostrini, K. Moriarty, and C. Rebbi, Phys. Rev.
D36, 3450 (1987).

[23] G. S. Bali, K. Schilling, and A. Wachter, Phys. Rev. D55, 5309 (1997), hep-lat/9611025.

[24] G. S. Bali, K. Schilling, and A. Wachter, Phys. Rev. D56, 2566 (1997), hep-lat/9703019.

[25] Y. Koike, Phys. Lett. B216, 184 (1989).

[26] K. Born, E. Laermann, T. Walsh, and P. Zerwas, Phys. Lett. B329, 332 (1994).

[27] Y. Koma and M. Koma, Nucl. Phys. B769, 79 (2007), hep-lat/0609078.

[28] Y. Koma and M. Koma, Prog. Theor. Phys. Suppl. 186, 205 (2010).

[29] N. Ishii, S. Aoki, and T. Hatsuda, Phys. Rev. Lett. 99, 022001 (2007), nucl-th/0611096.

[30] S. Aoki, T. Hatsuda, and N. Ishii, Prog. Theor. Phys. 123, 89 (2010), hep-lat/0909.5585.

[31] T. Kawanai and S. Sasaki, Phys. Rev. Lett. 107, 091601 (2011), hep-lat/1102.3246.

[32] H. Nemura, N. Ishii, S. Aoki, and T. Hatsuda, Phys. Lett. B673, 136 (2009), nucl-th/0806.1094.

[33] Y. Ikeda (HAL QCD Collaboration), Prog. Theor. Phys. Suppl. 186, 228 (2010).

[34] T. Kawanai and S. Sasaki, Phys. Rev. D82, 091501 (2010), hep-lat/1009.3332.

[35] T. Doi et al. (HAL QCD Collaboration), Prog. Theor. Phys. 127, 723 (2012), hep-lat/1106.2276.

[36] S. Aoki et al. (HAL QCD Collaboration) (2012), heplat/1206.5088.

[37] K. Murano, N. Ishii, S. Aoki, and T. Hatsuda, Prog. Theor. Phys. 125, 1225 (2011), hep-lat/1103.0619.

[38] S. Aoki et al. (HAL QCD Collaboration), Proc. Japan Acad. B87, 509 (2011), hep-lat/1106.2281.

[39] N. Ishii et al. (HAL QCD Collaboration), Phys. Lett. B712, 437 (2012), hep-lat/1203.3642.

[40] T. Kawanai and S. Sasaki, Phys. Rev. D85, 091503 (2012), hep-lat/1110.0888.

[41] B. Velikson and D. Weingarten, Nucl. Phys. B249, 433 (1985). 
[42] R. Gupta, D. Daniel, and J. Grandy, Phys. Rev. D48, 3330 (1993), hep-lat/9304009.

[43] W. E. Caswell and G. P. Lepage, Phys. Rev. A18, 810 (1978).

[44] Y. Ikeda and H. Iida, Prog.Theor.Phys. 128, 941 (2012), 1102.2097.

[45] K. Murano, N. Ishii, S. Aoki, and T. Hatsuda, PoS LAT2009, 126 (2009), hep-lat/1003.0530.

[46] M. Lüscher, Nucl. Phys. B354, 531 (1991).

[47] R. Sommer, Nucl. Phys. B411, 839 (1994), heplat/9310022.

[48] S. Aoki, Y. Kuramashi, and S.-i. Tominaga, Prog. Theor. Phys. 109, 383 (2003), hep-lat/0107009.

[49] A. X. El-Khadra, A. S. Kronfeld, and P. B. Mackenzie,
Phys. Rev. D55, 3933 (1997), hep-lat/9604004.

[50] N. H. Christ, M. Li, and H.-W. Lin, Phys. Rev. D76, 074505 (2007), hep-lat/0608006.

[51] H.-W. Lin and N. Christ, Phys. Rev. D76, 074506 (2007), hep-lat/0608005.

[52] Y. Aoki et al. (RBC Collaboration, UKQCD Collaboration) (2012), hep-lat/1206.2554.

[53] J. Beringer et al. (Particle Data Group), Phys. Rev. D86, 010001 (2012).

[54] Y. Kayaba et al. (CP-PACS Collaboration), JHEP 0702, 019 (2007), hep-lat/0611033.

[55] S. Gusken, Nucl.Phys.Proc.Suppl. 17, 361 (1990).

[56] Y. Sumino, in private communication. 